\title{
Formation of Jets and Equatorial Superrotation on Jupiter
}

\author{
TAPIO SCHNEIDER AND JUNJUN LIU \\ California Institute of Technology, Pasadena, California
}

(Manuscript received 31 March 2008, in final form 24 September 2008)

\begin{abstract}
The zonal flow in Jupiter's upper troposphere is organized into alternating retrograde and prograde jets, with a prograde (superrotating) jet at the equator. Existing models posit as the driver of the flow either differential radiative heating of the atmosphere or intrinsic heat fluxes emanating from the deep interior; however, they do not reproduce all large-scale features of Jupiter's jets and thermal structure. Here it is shown that the difficulties in accounting for Jupiter's jets and thermal structure resolve if the effects of differential radiative heating and intrinsic heat fluxes are considered together, and if upper-tropospheric dynamics are linked to a magnetohydrodynamic (MHD) drag that acts deep in the atmosphere and affects the zonal flow away from but not near the equator. Baroclinic eddies generated by differential radiative heating can account for the offequatorial jets; meridionally propagating equatorial Rossby waves generated by intrinsic convective heat fluxes can account for the equatorial superrotation. The zonal flow extends deeply into the atmosphere, with its speed changing with depth, away from the equator up to depths at which the MHD drag acts. The theory is supported by simulations with an energetically consistent general circulation model of Jupiter's outer atmosphere. A simulation that incorporates differential radiative heating and intrinsic heat fluxes reproduces Jupiter's observed jets and thermal structure and makes testable predictions about as yet unobserved aspects thereof. A control simulation that incorporates only differential radiative heating but not intrinsic heat fluxes produces off-equatorial jets but no equatorial superrotation; another control simulation that incorporates only intrinsic heat fluxes but not differential radiative heating produces equatorial superrotation but no offequatorial jets. The proposed mechanisms for the formation of jets and equatorial superrotation likely act in the atmospheres of all giant planets.
\end{abstract}

\section{Introduction}

The zonal flow in Jupiter's upper troposphere has been inferred by tracking cloud features, which move with the horizontal flow in the layer between about 0.5 and 1 bar atmospheric pressure (Ingersoll et al. 2004; West et al. 2004; Vasavada and Showman 2005). In this layer, the zonal flow is organized into a strong prograde (superrotating) equatorial jet and an alternating sequence of retrograde and prograde off-equatorial jets (Fig. 1a). This flow pattern has been stable at least between the observations by the Voyager and Cassini spacecrafts in 1979 and 2000, with some variations in jet speeds, for example, a slowing of the prograde jet at $21^{\circ} \mathrm{N}$ planetocentric latitude by $\sim 40 \mathrm{~m} \mathrm{~s}^{-1}$ (Porco et al. 2003; Ingersoll et al. 2004). The zonal flow in layers above the

Corresponding author address: Tapio Schneider, California Institute of Technology, 1200 E. California Blvd., Pasadena, CA 91125-2300.

E-mail: tapio@caltech.edu clouds has been inferred from the thermal structure of the atmosphere, using the thermal wind relation between meridional temperature gradients and vertical shears of the zonal flow. Meridional temperature gradients and thus vertical shears between 0.1 and 0.5 bar are generally small: meridional temperature contrasts along isobars do not exceed $\sim 10 \mathrm{~K}$ (Conrath et al. 1998; Simon-Miller et al. 2006). The thermal stratification in the same layer is statically stable (Simon-Miller et al. 2006; Read et al. 2006). About the zonal flow in lower layers, it is only known that at one site at $6.4^{\circ} \mathrm{N}$ planetocentric latitude, where the Galileo probe descended into Jupiter's atmosphere, it is prograde and increases with depth from $\sim 90 \mathrm{~m} \mathrm{~s}^{-1}$ at 0.7 bar to $\sim 170 \mathrm{~m} \mathrm{~s}^{-1}$ at 4 bar; beneath, it is relatively constant up to at least $\sim 20$ bar (Atkinson et al. 1998). At the same site, the thermal stratification is statically stable but approaches neutrality with increasing depth between 0.5 and 1.7 bar; beneath, it is statically nearly neutral or neutral up to at least $\sim 20$ bar (Magalhães et al. 2002). These are the large-scale features (if the Galileo probe data are 


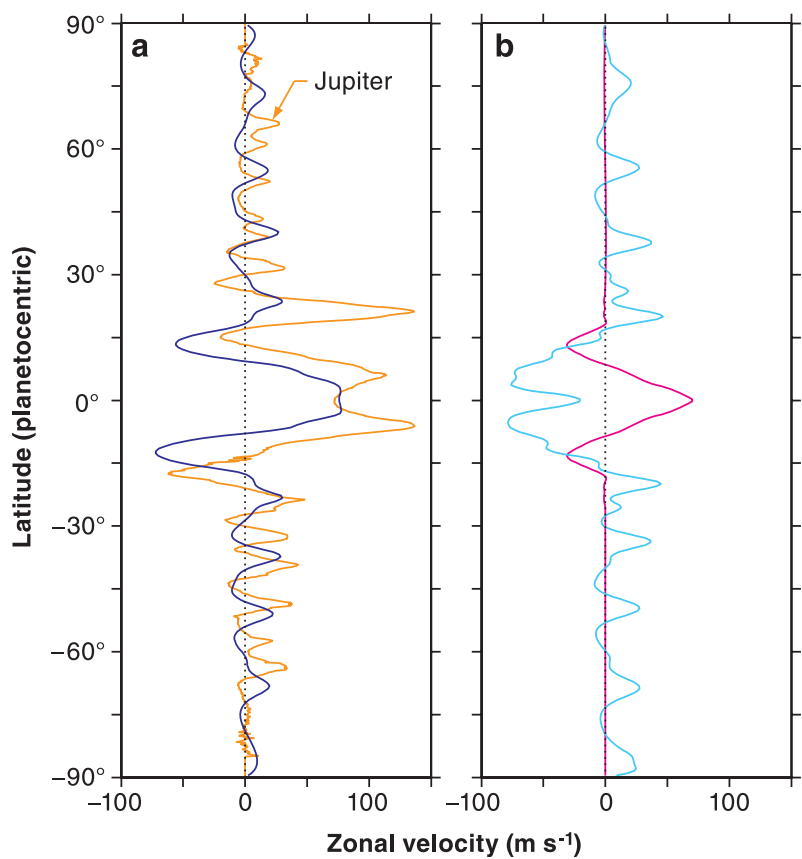

FIG. 1. Zonal flow in Jupiter's upper troposphere and in simulations. (a) Zonal velocity on Jupiter, inferred by tracking cloud features from the Cassini spacecraft (Porco et al. 2003) (orange) and in Jupiter simulation at 0.65 bar (blue). (b) Zonal velocity at 0.65 bar in control simulations: with intrinsic heat fluxes but with uniform insolation at the top of the atmosphere (magenta), and with differential insolation but without intrinsic heat fluxes (light blue). Zonal velocities in simulations are zonal and temporal means in statistically steady states (over 1500 days for Jupiter simulation and over 900 days for control simulations); differences between the (statistically identical) hemispheres here and in subsequent figures are indicative of sampling variability. For Jupiter, latitude here and throughout this paper is planetocentric; the simulated planets are spherical, so planetocentric and planetographic latitudes are identical.

representative of large scales) that a minimal model of Jupiter's general circulation should be able to reproduce.

There are two plausible energy sources for Jupiter's general circulation. First, $\sim 8 \mathrm{~W} \mathrm{~m}^{-2}$ of solar radiation are absorbed in Jupiter's atmosphere (Hanel et al. 1981), where the time-mean insolation at the top of the atmosphere, given Jupiter's small obliquity of $3^{\circ}$, varies approximately with the cosine of latitude. Second, $\sim 6 \mathrm{~W} \mathrm{~m}^{-2}$ of intrinsic heat fluxes emanate from Jupiter's deep interior (Ingersoll et al. 2004; Guillot et al. 2004; Guillot 2005); observations of convective storms (Gierasch et al. 2000; Porco et al. 2003; Sánchez-Lavega et al. 2008) and the neutral or nearly neutral thermal stratification along much of the Galileo probe descent path show that the intrinsic heat fluxes are at least partially convective. Existing models of Jupiter's zonal flow posit as the driver of the general circulation either differential radiative heating of the atmosphere or intrinsic convective heat fluxes (e.g., Busse 1976, 1994; Williams 1979; Ingersoll et al. 2004; Vasavada and Showman 2005); however, they do not reproduce all large-scale features of the jets and thermal structure. For example, in parameter regimes relevant for Jupiter, they generally do not produce equatorial superrotation unless artifices are employed such as imposing an additional heat source near the equator (Williams 2003) or assuming excessively viscous flow and permitting intrinsic heat fluxes several orders of magnitude stronger than Jupiter's (Heimpel et al. 2005; Heimpel and Aurnou 2007).

Here we show that the difficulties in accounting for Jupiter's jets and thermal structure resolve if the effects of differential radiative heating and intrinsic heat fluxes are considered together, and if upper-tropospheric dynamics are linked to drag that acts deep in the atmosphere and affects the zonal flow away from but not near the equator. The key is to distinguish the different ways in which eddies can be generated near the equator and away from it and to consider their role and the role of drag at depth in the balance of angular momentum (the angular momentum component about the planet's spin axis).

First we describe how eddies can be generated near the equator and away from it, how convectively generated equatorial waves can lead to equatorial superrotation, and how the angular momentum balance of the upper troposphere is linked to drag and constrains the flow at depth (sections 2-4). Then we use simulations with a three-dimensional general circulation model (GCM) of a thin shell in Jupiter's outer atmosphere, with an idealized representation of effects of drag deep in the atmosphere, to demonstrate that the mechanisms proposed can account for Jupiter's observed jets and thermal structure (sections 5-7).

\section{Eddy generation and angular momentum fluxes}

Tracking of cloud features shows that eddies in Jupiter's upper troposphere transport angular momentum meridionally from retrograde into prograde jets; the eddy angular momentum fluxes extend at least over the layer between about 0.5 and 1 bar and imply there a mean conversion rate from eddy to mean flow kinetic energy per unit of mass of order $10^{-5}$ to $10^{-4} \mathrm{~W} \mathrm{~kg}^{-1}$ (Ingersoll et al. 1981; Salyk et al. 2006). If the eddy angular momentum fluxes extended unabatedly over a layer more than tens of bars thick, the total conversion rate from eddy to mean flow kinetic energy would exceed the rate at which the atmosphere takes up energy from 
solar radiation and intrinsic heat fluxes combined. For example, if the eddy angular momentum fluxes extended over a layer of 2.5 bar thickness, and if vertical variations of the zonal flow over this layer are negligible, the total conversion rate would already be of order $1 \mathrm{~W} \mathrm{~m}^{-2}$ - that is, it would amount to $\sim 5 \%$ of the energy uptake by the atmosphere (Salyk et al. 2006). But probably only a small fraction of the atmosphere's energy uptake is available to generate eddy kinetic energy. This means that columnar-flow models of Jupiter's general circulation, in which the momentum exchange between eddies and the mean flow extends over layers with thickness of order $10^{6}$ bar and greater, are not viable on energetic grounds: they require energy sources several orders of magnitude larger than Jupiter's (e.g., Sun et al. 1993; Heimpel et al. 2005; Heimpel and Aurnou 2007). It also means that the thin-shell approximation -in which the distance from any point in the atmosphere to the planet's center is taken to be constant and equal to the planetary radius-is adequate for and will be made in the following considerations of the tropospheric eddy transport of angular momentum (although the zonal flow can extend deeply; see section 4).

Meridional eddy transport of angular momentum is evidence of meridional eddy propagation and irreversible dynamics (e.g., Edmon et al. 1980). In a thin shell, eddies that propagate meridionally transport angular momentum in the opposite direction of their propagation if the meridional gradient of absolute vorticity, or depth-averaged potential vorticity, is positive (northward) between their generation and dissipation (breaking) regions. In the dissipation regions, irreversible meridional mixing of absolute vorticity then leads to southward eddy vorticity fluxes and divergence of meridional eddy angular momentum fluxes; the compensating convergence of meridional eddy angular momentum fluxes occurs in the generation regions, implying angular momentum transport from the dissipation into the generation regions (Kuo 1951; Held 1975; Andrews and McIntyre 1976, 1978; Rhines 1994; Vallis 2006, chapter 12). In Jupiter's upper troposphere, the meridional gradient of absolute vorticity (and potential vorticity) is generally positive, except in narrow latitude bands at the centers of retrograde jets (Ingersoll et al. 1981; Read et al. 2006). The direction of the observed eddy angular momentum fluxes hence indicates generation of eddies in prograde jets, dissipation in retrograde jets, and propagation in between.

In prograde off-equatorial jets, eddies can be generated by baroclinic instability. Prograde jets are baroclinically more unstable than retrograde jets if the speed (absolute value of velocity) of the zonal flow in prograde jets decreases with depth and in retrograde jets either also decreases with depth or, with weaker vertical shear, increases with depth. By thermal wind balance, meridional temperature gradients along isobars then are equatorward in prograde jets and either are also equatorward but weaker or are poleward in retrograde jets. Indeed, in Jupiter's upper troposphere (at $\sim 0.25$ bar), meridional temperature gradients have been observed to be equatorward in prograde off-equatorial jets and either equatorward but weaker or poleward in retrograde off-equatorial jets [compare Fig. 6 in Simon-Miller et al. (2006) to Fig. 3 in Vasavada and Showman (2005)]. Thus, meridional propagation of eddies generated by baroclinic instability preferentially in prograde jets can account for the angular momentum transport from retrograde into prograde off-equatorial jets, as demonstrated in baroclinic GCMs that produce multiple jets (e.g., Williams 1979; O'Gorman and Schneider 2008).

In the prograde equatorial jet, eddies can be generated by intrinsic convective heat fluxes. Unlike in higher latitudes, Coriolis forces are small in the equatorial region, where the Rossby number is order one or greater. Horizontal pressure gradients there are limited by the necessity to be balanced primarily by inertial accelerations, rather than or in addition to Coriolis accelerations. Hence, they are small (of order Froude number), and so are horizontal temperature gradients on scales large enough that hydrostatic balance holds (Charney 1963). ${ }^{1}$ On such large scales, therefore, diabatic heating $Q$ cannot be balanced by temperature fluctuations or horizontal temperature advection, as in higher latitudes. Instead, as in the tropics of Earth's atmosphere, it is primarily balanced by the adiabatic cooling associated with vertical motion $\omega$ acting on a (possibly small) static stability $S$, giving the weak temperature gradient approximation

\footnotetext{
${ }^{1}$ The angular momentum and hydrostatic equations imply that, near the equator, horizontal variations in pressure $p$ and potential temperature $\theta$ scale as $\delta p / p \sim \delta \theta / \theta \sim \mathrm{Fr}$, where $\mathrm{Fr}=U V /(g H)$ is a Froude number, $H$ is the scale height, $U$ is a mean zonal velocity scale, and $V$ is the greater of an eddy velocity scale or mean meridional velocity scale (cf. Charney 1963). For Jupiter parameters and with $H \sim 20 \mathrm{~km}, U \sim 100 \mathrm{~m} \mathrm{~s}^{-1}$, and $V \sim 10 \mathrm{~m} \mathrm{~s}^{-1}$ (Porco et al. 2003; Salyk et al. 2006), one obtains Fr $\sim 10^{-3}$. This scaling holds where the Rossby number Ro $=U /|f L|$, with length scale of flow variations $L$, is order one or greater. With $f=\beta y$ and $L \lesssim|y|$, it follows that the scaling holds at least within meridional distances $|y| \sim(U / \beta)^{1 / 2}$ of the equator (Sobel et al. 2001), which, for Jupiter, is within $\sim 5000 \mathrm{~km}$ or $\sim 4^{\circ}$ of the equator.
} 
of the thermodynamic equation ${ }^{2}$ (Held and Hoskins 1985; Sobel et al. 2001)

$$
\omega S \approx-Q .
$$

By mass conservation, $\nabla_{h} \cdot \mathbf{v}_{\chi}=-\partial_{p} \omega$, vertical gradients of diabatic heating and/or static stability thus induce large-scale horizontal divergence

$$
\nabla_{h} \cdot \mathbf{v}_{\chi} \approx \partial_{p}(Q / S)
$$

where we have decomposed the horizontal velocity $\mathbf{v}=$ $\mathbf{v}_{\chi}+\mathbf{v}_{\Psi}$ into divergent $\left(\mathbf{v}_{\chi}\right)$ and rotational $\left(\mathbf{v}_{\Psi}\right)$ components. As discussed by Sardeshmukh and Hoskins (1988), this horizontal divergence drives a rotational flow: Vortex stretching and vorticity advection by the divergent flow are a source

$$
R=-\zeta_{a}\left(\nabla_{h} \cdot \mathbf{v}_{\chi}\right)-\left(\mathbf{v}_{\chi} \cdot \nabla_{h}\right) \zeta_{a}
$$

of rotational flow, as can be seen from the equation for the absolute vorticity $\zeta_{a}=f+\mathbf{k} \cdot\left(\nabla_{h} \times \mathbf{v}_{\Psi}\right)=f+\zeta$ in the equatorial troposphere in the form

$$
\left(\partial_{t}+\mathbf{v}_{\Psi} \cdot \nabla_{h}\right) \zeta_{a} \approx R .
$$

Neglected here is the baroclinic term, consistent with the weak temperature gradient approximation (Charney 1963; Sobel et al. 2001), as well as friction and the vertical advection and tilting terms. It follows that convective heating fluctuations that cannot be balanced by slow radiative processes induce fluctuations in the large-scale horizontal divergence, and these represent a source

$$
R^{\prime}=R-\bar{R}=-\nabla_{h} \cdot\left(\zeta_{a} \mathbf{v}_{\chi}-\overline{\zeta_{a} \mathbf{v}_{\chi}}\right)
$$

of vorticity fluctuations and thus a source of Rossby waves (Sardeshmukh and Hoskins 1988). (Overbars denote isobaric zonal and temporal means and primes deviations therefrom.)

\footnotetext{
${ }^{2}$ We use pressure coordinates, with $S=-\partial_{p} \theta$ and $\omega=D p / D t$. To obtain the relative magnitudes of the terms on the left-hand side of the thermodynamic equation $\left(\partial_{t}+\mathbf{v} \cdot \nabla_{h}\right) \theta-\omega S=Q$, with isobaric horizontal derivative operator $\nabla_{h}$, we assume that the explicit time derivative and horizontal advection terms scale as $(V / L) \delta \theta$, and the vertical advection term scales as $(V / L) \Delta \theta$. We have used mass conservation to relate vertical to horizontal velocity scales, and $\Delta \theta$ is a vertical potential temperature change over a scale height. The vertical advection term then dominates, and the weak temperature gradient approximation (1) is adequate, if vertical potential temperature changes satisfy $\Delta \theta / \theta \gg \delta \theta / \theta \sim$ $\mathrm{Fr} \sim 10^{-3}$. In Jupiter's equatorial troposphere, if one takes the thermal stratification along the Galileo probe descent path as representative, this is assured at least above 1.7 bar (Magalhães et al. 2002).
}

Horizontally, the Rossby wave source (5) can be expected to be large in the equatorial region because the Rossby number there is order one or greater, and largescale horizontal flow fluctuations induced by convective heating fluctuations are divergent at leading order. In contrast, the Rossby number in higher latitudes is small, large-scale horizontal flow fluctuations are nondivergent at leading order, and baroclinic generation of vorticity fluctuations, neglected in the Rossby wave source (5), can be important. Scale analysis ${ }^{3}$ suggests that the Rossby wave source (5) is largest in the prograde equatorial jet and in the strong prograde jet at $21^{\circ} \mathrm{N}$-that is, not only in the latitude band in which the Rossby number is order one or greater (within $\sim 4^{\circ}$ of the equator; see footnote 1), but in a slightly wider latitude band around the equator and in strong jets generally. Vertically, the Rossby wave source (5) should be largest in the upper troposphere, below the top of the convective outflows, where fluctuations in the large-scale horizontal divergence (2) can be expected to be largest: Convective heating fluctuations and their vertical gradients can be expected to be largest there, and the static stability has substantial vertical gradients (Magalhães et al. 2002), marking the transition from lower layers that are neutrally stratified by convection to upper layers that are more stably stratified because the stabilizing radiative heating from above begins to have an effect. The Rossby wave source (5), then, should be largest in Jupiter's equatorial upper troposphere if convective heating fluctuations there are sufficiently strong.

\section{Generation of equatorial superrotation}

By Hide's theorem, the eddy transport of angular momentum into the equatorial region observed in Jupiter's

\footnotetext{
${ }^{3}$ For the scale analysis, we use the eddy velocity scale $V \sim$ $10 \mathrm{~m} \mathrm{~s}^{-1}$ and the length scale of flow variations $L \sim 5000 \mathrm{~km}$ both for eddies and for mean fields (cf. footnote 1); we take the scales to be invariant with latitude, as indicated, for the velocity scale, by the Cassini image analysis of Salyk et al. (2006). Then, where Ro $\gtrsim$ 1 and if horizontal velocity fluctuations are divergent at leading order, the Rossby wave source owing to advection of planetary vorticity by the divergent flow is of order $R^{\prime} \sim \beta V \sim 5 \times 10^{-11} \mathrm{~s}^{-2}$; the Rossby wave source owing to stretching of planetary vorticity, evaluated at $4^{\circ}$ latitude, is of the same order, $R^{\prime} \sim|f V| / L \sim 5 \times$ $10^{-11} \mathrm{~s}^{-2}$. Where Ro $<1$, the divergent velocity is of order Ro $V$, and the Rossby wave source is of order $R^{\prime} \sim \operatorname{Ro}|f V| / L=U V / L^{2}$. This is of the same order $\left(R^{\prime} \sim 5 \times 10^{-11} \mathrm{~s}^{-2}\right)$ as the Rossby wave source near the equator where the mean zonal velocity scale is of order $U \sim 100 \mathrm{~m} \mathrm{~s}^{-1}$ (throughout the prograde equatorial jet and in the strong prograde jet at $21^{\circ} \mathrm{N}$; see Fig. 1a). At other latitudes, the mean zonal velocity scale $\left(U \lesssim 30 \mathrm{~m} \mathrm{~s}^{-1}\right)$ and the Rossby wave source $\left(R^{\prime} \lesssim 10^{-11} \mathrm{~s}^{-2}\right)$ are smaller, albeit only by $O(1)$ factors.
} 
upper troposphere is necessary for the existence of a prograde equatorial jet (Hide 1969; Schneider 1977; Saravanan 1993; Held 1999; Schneider 2006). To see how such angular momentum transport can come about, consider the eddy enstrophy equation implied by the vorticity equation (4),

$$
\partial_{t} \overline{\zeta^{\prime 2}} / 2+\overline{v_{\Psi}^{\prime} \zeta^{\prime}} \partial_{y} \bar{\zeta}_{a} \approx \overline{R^{\prime} \zeta^{\prime}}
$$

where $y=a \phi$ is the meridional coordinate with planetary radius $a$ and latitude $\phi$. Neglected here is the advection of eddy enstrophy by the eddies themselves, a triple correlation term that seems to be about an order of magnitude smaller in Jupiter's equatorial upper troposphere than the retained term $\overline{v_{\Psi}^{\prime} \zeta^{\prime}} \partial_{y} \bar{\zeta}_{a}{ }^{4}$ In a statistically steady state, upon division by $\partial_{y} \bar{\zeta}_{a} \neq 0$ and multiplication by the thin-shell approximation of the distance $r_{\perp}=a \cos \phi$ to the planet's spin axis, the eddy enstrophy equation becomes the wave activity balance (Andrews and McIntyre 1976, 1978; Edmon et al. 1980)

$$
G \approx \overline{v_{\Psi}^{\prime} \zeta^{\prime}} r_{\perp}=-\operatorname{div}\left(\overline{u_{\Psi}^{\prime} v_{\Psi}^{\prime}} r_{\perp}\right)
$$

Here, $\operatorname{div}(\cdot)$ is the isobaric meridional divergence operator, and

$$
G=\frac{\overline{R^{\prime} \zeta^{\prime}}}{\partial_{y} \zeta_{a}} r_{\perp}
$$

represents the generation of wave activity $A$, with

$$
A=\frac{1}{2} \frac{\overline{\zeta^{\prime 2}}}{\partial_{y} \bar{\zeta}_{a}} r_{\perp} .
$$

The wave activity balance (7) states that at latitudes at which wave activity is generated $(G>0)$, the eddy vorticity flux $\overline{v_{\Psi}^{\prime} \zeta^{\prime}}$ is directed northward, implying convergence of (rotational) eddy angular momentum fluxes $\overline{u_{\Psi}^{\prime} v_{\Psi}^{\prime}} r_{\perp}$ (per unit mass); conversely, at latitudes at which wave activity is dissipated $(G<0)$, there is divergence of eddy angular momentum fluxes. The meridional eddy angular momentum flux is equal to minus the meridional wave activity flux. Thus, generation of wave activity in the equatorial region and radiation to

\footnotetext{
${ }^{4}$ To estimate the relative magnitude of the terms, we roughly approximate the absolute vorticity gradient $\partial_{y} \bar{\zeta}_{a}$ by $\beta$ (Read et al. 2006) and use the horizontal length scale of flow variations $L \sim 5000 \mathrm{~km}$ (see footnote 3 ) and the rotational meridional eddy velocity scale $V \sim 10 \mathrm{~m} \mathrm{~s}^{-1}$ (Salyk et al. 2006); that is, we assume that the rotational and divergent velocities are of the same order (cf. footnote 3 ). Then, the magnitude of the neglected eddy enstrophy advection $\operatorname{div}\left(\overline{v_{\Psi}^{\prime} \zeta^{\prime 2}}\right) / 2$ relative to the retained term $\overline{v_{\Psi}^{\prime} \zeta^{\prime}} \partial_{y} \bar{\zeta}_{a}$ is of order $V /\left(\beta L^{2}\right) \sim 0.1$.
}

and dissipation in higher latitudes entail angular momentum transport from higher latitudes into the equatorial region. See Andrews and McIntyre (1976, 1978), Plumb (1979), McIntyre (1980), Edmon et al. (1980), and Vallis (2006, chapter 7) for further discussion and generalizations of these results from wave-mean flow interaction theory; and see Suarez and Duffy (1992) and Saravanan (1993) for numerical demonstrations that (stationary) Rossby wave sources in the equatorial region entail convergence of eddy angular momentum fluxes and can generate superrotation.

The wave activity balance (7) implies that in Jupiter's upper troposphere, convergence of eddy angular momentum fluxes in the equatorial region is to be expected if Rossby wave generation by convective heating fluctuations is sufficiently strong. Contributions to the Rossby wave source $R^{\prime}$ that do not depend on vorticity fluctuations $\zeta^{\prime}$, to the extent that $\partial_{t} \zeta^{\prime} \sim R^{\prime}$, can be expected to contribute positively to the eddy enstrophy generation $\overline{R^{\prime} \zeta^{\prime}}$ and thus, because the absolute vorticity gradient is positive (Read et al. 2006), to the wave activity generation $G$. Some of the wave activity so generated may dissipate near or in its generation region because contributions to the Rossby wave source $R^{\prime}$ that depend on vorticity fluctuations $\zeta^{\prime}$ may damp them. The principal damping term is $-\left(\operatorname{div} \bar{v}_{\chi}\right) \overline{\zeta^{\prime 2}}$; as pointed out by Sardeshmukh and Hoskins (1988), this term contributes negatively to the eddy enstrophy and wave activity generation where the mean meridional flow is divergent, for example, in the horizontal outflows of ascending branches of any (Eulerian) mean meridional circulation cells. Near the equator, however, the damping limits itself as the strength of convective heating fluctuations increases: wave activity generation near the equator entails convergence of eddy angular momentum fluxes, which implies weakened poleward or even equatorward mean meridional flow, as can be seen from the zonally and temporally averaged angular momentum balance in a statistically steady state,

$$
-\overline{\mathbf{u}} \cdot \nabla M_{\Omega}=f \bar{v}_{\chi} r_{\perp}=\mathcal{S}_{e}+\mathcal{S}_{m} .
$$

Here, $\mathbf{u}$ is the three-dimensional velocity vector, $M_{\Omega}=$ $\Omega r_{\perp}^{2}$ is the angular momentum per unit mass owing to the planetary rotation (with constant angular velocity $\Omega$ ), and the first equality holds in the thin-shell approximation. We have again neglected friction; we have used the fact that the mean meridional flow is irrotational $\left(\bar{v}=\bar{v}_{\chi}\right)$; and

$$
\begin{aligned}
\mathcal{S}_{e} & =\operatorname{div}\left(\overline{u^{\prime} v^{\prime}} r_{\perp}\right)+\partial_{p}\left(\overline{u^{\prime} \omega^{\prime}} r_{\perp}\right) \quad \text { and } \\
\mathcal{S}_{m} & =\operatorname{div}\left(\bar{u} \bar{v} r_{\perp}\right)+\partial_{p}\left(\bar{u} \bar{\omega} r_{\perp}\right),
\end{aligned}
$$


are the divergences of fluxes of relative angular momentum per unit mass $M_{u}=u r_{\perp}$ owing to eddies and mean meridional circulations. The angular momentum balance (10) shows that as the strength of convective heating fluctuations increases, increasing wave activity generation and the increasing eddy angular momentum flux convergence (decreasing $\mathcal{S}_{e}$ ) it entails either weakens any divergence of the mean meridional flow at the equator or even leads to convergence and thus to eddy enstrophy and wave activity amplification. ${ }^{5}$ Sufficiently strong convective heating fluctuations, then, can be expected to lead to net wave activity generation $(G>0)$ and convergence of eddy angular momentum fluxes in the equatorial region.

Convergence of eddy angular momentum fluxes in the equatorial region accelerates the zonal flow. As a consequence, a prograde equatorial jet forms if other processes that may decelerate it-e.g., drag at depth linked to upper-tropospheric dynamics through mean meridional circulations (see section 4)-are sufficiently weak. Such an equatorial jet can be expected to occupy a latitude band at least as wide as the meridional decay scale of long equatorial Rossby waves: the equatorial Rossby radius $L_{e}=(c / \beta)^{1 / 2}$ with tropospheric gravity wave speed $c$ (Matsuno 1966; Gill 1982, chapter 11). For Jupiter, if one takes the speed $c \approx 450 \mathrm{~m} \mathrm{~s}^{-1}$ of the waves observed after the impact of comet ShoemakerLevy 9 as the relevant gravity wave speed (Ingersoll and Kanamori 1995) — a wave speed roughly consistent with the tropospheric thermal stratification along the Galileo probe descent path (cf. Magalhães et al. 2002)—one obtains $L_{e} \approx 9500 \mathrm{~km}$, or a lower bound on the halfwidth of the jet of $\sim 8^{\circ}$. This is similar to, albeit smaller than, the half-width of Jupiter's equatorial jet in the upper troposphere (Fig. 1a). ${ }^{6}$

An upper bound on the speed of the equatorial jet can be obtained by vorticity homogenization arguments. The jet speed is determined by the change in vorticity within the equatorial jet owing to the meridional redistribution of absolute vorticity relative to a state of

\footnotetext{
${ }^{5}$ This is one of several eddy-mean flow feedbacks that can lead to rapid transitions to superrotation as parameters such as the strength of the convective heating are varied. Other feedbacks are discussed by Saravanan (1993) and Held (1999).

${ }^{6}$ Other lower bounds on the half-width of the equatorial jet are the half-widths of the latitude bands with (i) substantial fluctuations in the horizontal divergence or (ii) relative angular momentum transport by mean meridional circulations. Both latitude bands are characterized by Ro $\gtrsim 1$ (e.g., Sobel et al. 2001; Schneider 2006). With the upper bound $U \lesssim c / 2$ on the equatorial jet speed given below, one finds that the half-width of the latitude band with Ro $\gtrsim 1$ is constrained to be of the same order as but smaller than $L_{e}$ (cf. footnote 1 ).
}

solid body rotation. If the zonal flow is zero at a distance $L_{s}$ from the equator and if this distance is small enough that the small-angle approximation for latitudes is adequate, the jet speed at the equator is $U \approx \Delta \zeta L_{s}$, where $\Delta \zeta$ is the absolute value of the relative vorticity averaged between the equator and a distance $L_{s}$ away from it. The absolute vorticity redistribution reaches an end state, and meridional Rossby wave propagation ceases, when the absolute vorticity within the jet is homogenized in each hemisphere. If the absolute vorticity at the zeros of the jet is approximated by $\pm \beta L_{s}$, the state of homogenized absolute vorticity corresponding to maximum prograde jet speed for a given jet width has absolute vorticity $+\beta L_{s}$ in the northern and $-\beta L_{s}$ in the Southern Hemisphere, with a barotropically stable jump at the equator: an absolute vorticity "staircase" (McIntyre 1982; Dritschel and McIntyre 2008). The absolute value of the relative vorticity averaged between the equator and a distance $L_{s}$ away from it then is $\Delta \zeta \approx \beta L_{s} / 2$, and one obtains the upper bound on the jet speed

$$
U \lesssim \frac{\beta L_{s}^{2}}{2}
$$

If one further substitutes $L_{s} \sim L_{e}$, one obtains $U \lesssim c / 2$, that is, a bound on the jet speed that only depends on the gravity wave speed. For Jupiter, with $c \approx 450 \mathrm{~m} \mathrm{~s}^{-1}$, this gives $U \lesssim 225 \mathrm{~m} \mathrm{~s}^{-1}$, which is of the same order as, albeit larger than, the observed equatorial jet speed in the upper troposphere (Fig. 1a); it is closer to the equatorial jet speed at greater depth along the Galileo probe descent path. If the actual half-width of Jupiter's equatorial jet is used $\left(L_{s} \gtrsim L_{e}\right)$, the overestimation of the jet speed in the upper troposphere is more pronounced; a state of homogenized absolute vorticity in each hemisphere is not attained in the upper troposphere (Read et al. 2006). Nonetheless, these arguments show that the speed of prograde equatorial jets, to the extent that a state of homogenized absolute vorticity in each hemisphere is being approached, can be expected to increase roughly linearly with $\beta$ and quadratically with their half-width. The speed of such jets does not depend directly, as is sometimes surmised, on the total energy uptake by the atmosphere or the total kinetic energy dissipation, but on emergent properties such as the thermal stratification, which determines the gravity wave speed. Consistent with these arguments and the similar radii and rotation rates of Jupiter and Saturn, Saturn's prograde equatorial jet is about 2 times wider and 3-4 times stronger than Jupiter's. This may be a consequence of a greater gravity wave speed on Saturn. 


\section{Drag at depth and mean meridional circulations}

In a statistically steady state, eddy angular momentum transport from retrograde into prograde jets in the upper troposphere must be balanced by angular momentum transport by mean meridional circulations or eddies in other layers or by drag on the zonal flow. Drag acts on the zonal flow deep in Jupiter's atmosphere, where hydrogen, its primary constituent, undergoes a continuous transition from an electrically semiconducting molecular phase in the outer layers to a conducting metallic phase in the interior (Guillot et al. 2004; Guillot 2005). Where the atmosphere is electrically conducting, any flow advects the magnetic field and induces an electric current. The Ohmic dissipation of the induced current implies a magnetohydrodynamic (MHD) drag on the flow (Grote and Busse 2001; Liu et al. 2008). This MHD drag we take to provide the principal angular momentum dissipation mechanism on Jupiter. Because the electric conductivity increases continuously with depth, it is difficult to estimate where in the atmosphere the MHD drag acts. For the sake of argument here, we assume that substantial MHD drag is confined within $\sim 0.96$ Jupiter radii (pressures greater than $\sim 10^{5}$ bar). This corresponds to the estimated maximum depth up to which zonal flows with speeds similar to those in the upper troposphere could extend before the energy dissipation by the MHD drag would violate the constraint that it cannot exceed Jupiter's luminosity (Liu et al. 2008). Precisely at which depth the MHD drag acts is not essential for our arguments, but it is crucial that there is an outer atmospheric shell in which the flow is effectively frictionless.

Because the MHD drag acts at great depth, deepatmosphere dynamics must be considered in linking it to dynamics in the upper troposphere. It is well known that in a thin atmospheric shell, in the zonal, temporal, and vertical mean in a statistically steady state, drag on the zonal flow balances any transport of angular momentum into or out of a latitude band (e.g., Peixoto and Oort 1992, chapter 11); that is, the zonally, temporally, and vertically averaged angular momentum balance is

$$
\left\langle\mathcal{S}_{e}+\mathcal{S}_{m}\right\rangle=r_{\perp}\langle\overline{\mathcal{D}}\rangle,
$$

where $\langle\cdot\rangle$ denotes a mass-weighted vertical mean and $\mathcal{D}$ is the drag force per unit mass on the zonal flow; for example, $\mathcal{D}=-k u$ with relaxation coefficient $k$ for Rayleigh drag. This generalizes to a deep atmosphere if $r_{\perp}=r \cos \phi$ is taken to be the actual distance to the planet's spin axis, with distance to the planet's center $r$, and if the vertical mean is understood as a mean along surfaces of constant planetary angular momentum per unit mass $M_{\Omega}=\Omega r_{\perp}^{2}$. Such angular momentum surfaces are vertical in the thin-shell approximation
( $r=a=$ const) but are parallel to the planet's spin axis in a deep atmosphere. That is, the zonal and vertical mean in a thin atmosphere must be replaced by a mean along cylinders concentric with the planet's spin axis in a deep atmosphere (see appendix A).

In the equatorial region in which cylinders concentric with the planet's spin axis do not intersect the layer with MHD drag, there is effectively (absent other dissipation mechanisms except weak viscous dissipation) no cylindrically averaged drag $\langle\overline{\mathcal{D}}\rangle$ on the zonal flow. If substantial MHD drag is confined within $\sim 0.96$ Jupiter radii, the region of vanishing cylindrically averaged MHD drag extends in the outer atmosphere from the equator to $\sim 16^{\circ}$ latitude in each hemisphere and projects from there downward along cylinders (see the schematic in Fig. 2). In this region, the angular momentum balance (13) implies that any convergence of eddy angular momentum fluxes in the upper troposphere can only be balanced by divergence of eddy angular momentum fluxes in other layers and/or by divergence of relative angular momentum fluxes owing to mean meridional circulations. The mean meridional circulations likely cannot link equatorial convergence of eddy angular momentum fluxes in the upper troposphere to the MHD drag at depth, as considerations of Rossby numbers show. Mean flows in the meridional direction can only lead to substantial divergence of relative angular momentum fluxes where the Rossby number Ro $=U /|f L|$, with meridional length scale of zonal-flow variations $L$, is order one or greater. The region where Ro $\gtrsim 1$ has a meridional half-width $\leqslant L_{e}$ (see footnote 6), even at depths at which the zonal flow speed may exceed its upper-tropospheric value but remains constrained by the bound (12). It extends to $\leqslant 8^{\circ}$ latitude in the outer atmosphere, so the latitude band in which $\mathrm{Ro} \geq 1$ is narrower than the latitude band of vanishing cylindrically averaged MHD drag, provided there is no MHD drag in an outer atmospheric shell at least $\sim 0.01$ Jupiter radii thick. Similarly, mean flows in the cylindrically radial direction (perpendicular to the planet's spin axis) can only lead to substantial divergence of relative angular momentum fluxes where the Rossby number $\mathrm{Ro}_{\perp}=U /\left(2 \Omega L_{\perp}\right)$, with cylindrically radial length scale of zonal-flow variations $L_{\perp}$, is order one or greater (see appendix A). With velocity scale $U \sim 200 \mathrm{~m} \mathrm{~s}^{-1}$ and length scale $L_{\perp} \sim 2000 \mathrm{~km}$, corresponding to the radial length scale in the equatorial plane of a jet that extends from the equator to $\sim 13^{\circ}$ latitude in the outer atmosphere and projects downward along cylinders, this Rossby number is $\mathrm{Ro}_{\perp} \sim 0.3$; it is even smaller in higher latitudes where the zonal flow velocity must be smaller because it cannot substantially exceed its upper-tropospheric values of order $10 \mathrm{~m} \mathrm{~s}^{-1}$ without violating the constraint that the energy dissipation by the MHD drag cannot 


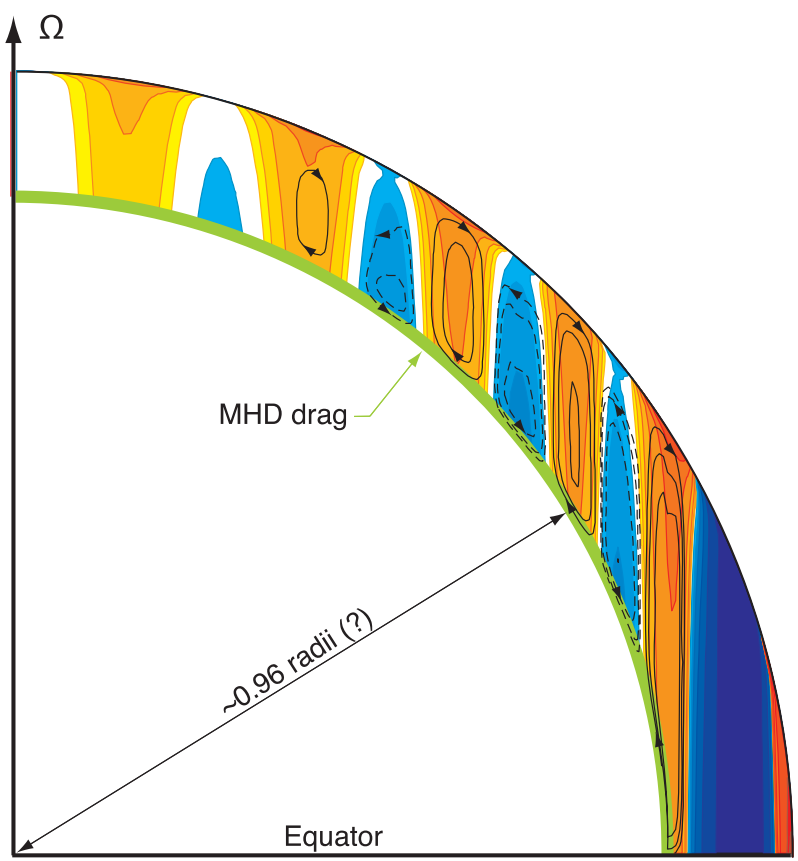

FIG. 2. Schematic of Jupiter's envisaged zonal flow and mean meridional circulations. Shown is one quadrant in the meridional plane (not to scale). Colors indicate the zonal flow (yellow/red for prograde and cyan/blue for retrograde flow). Contours indicate the mass flux streamfunction of the mean meridional circulation (solid for clockwise rotation and dashed for counterclockwise rotation). We have omitted mean meridional circulations in the equatorial region in which cylinders concentric with the planet's spin axis do not intersect the layer with MHD drag; they likely have a more complex structure than those in higher latitudes.

exceed Jupiter's luminosity (Liu et al. 2008). Therefore, mean meridional circulations can redistribute angular momentum only within a region that is narrower than that in which cylinders concentric with the planet's spin axis do not intersect the layer with MHD drag, provided there is no MHD drag in an outer atmospheric shell at least $\sim 0.01$ Jupiter radii thick. Thus, they likely cannot link equatorial convergence of eddy angular momentum fluxes resulting from meridional radiation of convectively generated Rossby waves to the MHD drag at depth. To the extent that divergence of eddy angular momentum fluxes outside the layer of convective Rossby wave generation and divergence of relative angular momentum fluxes owing to mean meridional circulations merely compensate the acceleration of the cylindrically averaged zonal flow, the generation of equatorial superrotation by Rossby wave radiation seems inevitable.

In higher latitudes, where cylinders concentric with the planet's spin axis do intersect the layer with MHD drag, mean meridional circulations link dynamics in the upper troposphere to the MHD drag at depth. The Rossby numbers $\mathrm{Ro}$ and $\mathrm{Ro}_{\perp}$ in higher latitudes are small, both in the upper troposphere and in deeper layers. Therefore, the relative angular momentum flux divergence owing to eddies dominates that owing to mean meridional circulations, and the local angular momentum balance (10) in the upper troposphere, where there is effectively no drag on the zonal flow, reduces to

$$
f \bar{v}_{\chi} r_{\perp} \approx \mathcal{S}_{e} .
$$

It follows that in regions of eddy angular momentum flux convergence (prograde jets), the mean meridional mass flux is equatorward; in regions of divergence (retrograde jets), it is poleward. Between the layer with substantial eddy angular momentum fluxes and the layer with MHD drag, provided that the divergence of convective angular momentum fluxes (Reynolds stress) is negligible, the mean meridional circulations are unaffected by zonal torques $\left(S_{e} \approx r_{\perp} \mathcal{D} \approx 0\right)$; the local angular momentum balance (10) in a form suitable for a deep atmosphere can then be expressed as

$$
\overline{\mathbf{u}} \cdot \nabla \bar{M} \approx 0,
$$

where $M=M_{\Omega}+M_{u}$ is the angular momentum per unit mass. It follows that the mean meridional circulations extend downward along surfaces of constant angular momentum per unit mass $M$ (e.g., Haynes et al. 1991). Approximately, these angular momentum surfaces are again cylinders concentric with the spin axis because small Rossby numbers mean that the angular momentum $M$ is dominated by its planetary component $M_{\Omega}$. Irrespective of the depth at which the MHD drag acts, the mean meridional circulations must extend downward to and must close where the drag allows mass fluxes to cross angular momentum surfaces (Haynes et al. 1991; O'Gorman and Schneider 2008). There, the Coriolis torque on any mass flux component normal to angular momentum surfaces is balanced by the MHD drag on the zonal flow (Ekman balance),

$$
\overline{\mathbf{u}} \cdot \nabla M_{\Omega} \approx r_{\perp} \mathcal{D} .
$$

Figure 2 sketches the resulting mean meridional circulations.

Ekman balance (16) at depth requires that the zonal flow is prograde $(\mathcal{D}<0)$ where the mean meridional mass flux has a component toward the spin axis and retrograde $(\mathcal{D}>0)$ where it has a component away from the spin axis. Given the correlation between the zonal velocity and the convergence of eddy angular momentum fluxes in the upper branches of the mean meridional circulations, this implies that the signs and zeros of the zonal flow, like the mean meridional circulations, project downward approximately along cylinders, as sketched 
in Fig. 2. Thermal wind balance then constrains the thermal structure of the atmosphere below the layer with substantial eddy angular momentum fluxes [see, e.g., Ingersoll and Pollard (1982) and Kaspi (2008) for thermal wind equations for deep atmospheres]. The mean meridional circulations adjust entropy gradients and the zonal flow in lower layers such that they satisfy, in a statistically steady state, the constraints that (i) angular momentum flux convergence or divergence and the MHD drag on the zonal flow balance upon averaging over cylinders, and (ii) the zonal flow is in thermal wind balance with the entropy gradients (see, e.g., Haynes et al. 1991). These dual constraints generally cannot be satisfied, as is often assumed in Jupiter models, with entropy gradients that vanish throughout the deep atmosphere and with a corresponding zonal flow without shear in the direction of the spin axis (Taylor columns). The zonal flow speed within $\sim 0.96$ Jupiter radii is constrained to be smaller than that in the prograde offequatorial jets in the upper troposphere because otherwise the energy dissipation by the MHD drag would exceed Jupiter's luminosity (Liu et al. 2008). Therefore, the zonal flow generally must be sheared and must be associated with nonzero entropy gradients. For example, it is conceivable that convection deep in Jupiter's atmosphere homogenizes entropy along angular momentum surfaces but that there are nonzero (albeit possibly weak) entropy gradients normal to them, such that the mean thermal structure at depth is neutral with respect to slantwise convective, or symmetric, instability.

\section{General circulation model}

To demonstrate the viability of the mechanisms proposed, we constructed a GCM of Jupiter's outer atmosphere. Simulating Jupiter's deep atmosphere in a manner that is consistent with its measured energy balance is computationally prohibitive. Instead, our GCM is based on the hydrostatic primitive equations for a dry ideal-gas atmosphere in a thin spherical shell with Jupiter's radius, rotation rate, gravitational acceleration, and thermal properties. The shell extends from the top of the atmosphere to a lower boundary with mean pressure 3 bar. Within this pressure range, about $90 \%$ of the solar radiation incident at the top of Jupiter's atmosphere is absorbed or scattered back, and latent heat release in phase changes of water, ammonia, and hydrogen sulfide is negligible: moist-adiabatic and dryadiabatic temperature lapse rates are nearly indistinguishable (Showman and Ingersoll 1998; Ingersoll et al. 2004). Nonetheless, the idealization of focusing on a thin shell in Jupiter's outer atmosphere means that we are not able to resolve details of the coupling between the flow in the outer atmosphere and that at depth, beyond the previously discussed constraints on this coupling implied by the angular momentum balance.

The GCM uses the spectral transform method in the horizontal (resolution T213) and finite differences in the vertical (30 levels). Radiative transfer is represented as that in a homogeneous gray atmosphere, with absorption and scattering of solar radiation and absorption and emission of thermal radiation. Optical properties of the atmosphere are so specified that the idealized representation of radiative transfer is qualitatively consistent with measured radiative fluxes inside Jupiter's atmosphere (Sromovsky et al. 1998) and quantitatively consistent with the measured energy balance at the top of the atmosphere (Hanel et al. 1981). At the GCM's lower boundary, a spatially uniform and temporally constant intrinsic heat flux is imposed. If the intrinsic heat flux is sufficiently strong to destabilize the atmospheric thermal stratification, the convection that ensues is represented by a quasi-equilibrium convection scheme that relaxes temperatures in statically unstable parts of atmospheric columns to a profile with neutral static stability (Schneider and Walker 2006). Near the lower boundary, Rayleigh drag in the horizontal momentum equations is chosen as an idealized linear representation of effects of the MHD drag deep in Jupiter's atmosphere on the flow in the outer atmosphere. To represent the downward projection along cylinders in a deep atmosphere in the thin-shell approximation with vertical planetary angular momentum surfaces, we use a Rayleigh drag coefficient that is constant along the GCM's lower boundary poleward of $16.3^{\circ}$ latitude and rapidly decreases to zero at lower latitudes, corresponding to the assumption that substantial MHD drag is confined within 0.96 Jupiter radii. Above the layer with Rayleigh drag, highly scale-selective horizontal hyperdiffusion at small scales (Smith et al. 2002), representing subgridscale processes, is the only frictional process; in particular, there is no vertical viscous transfer of momentum or heat. All forcings and boundary conditions are temporally constant and zonally and hemispherically symmetric, so the simulations have stationary and zonally and hemispherically symmetric flow statistics. See appendix B for details of the GCM and simulations.

\section{Jupiter simulation \\ a. Upper-tropospheric dynamics}

A simulation with Jupiter's solar constant, with insolation at the top of the atmosphere varying with the cosine of latitude, and with an intrinsic heat flux of $5.7 \mathrm{~W} \mathrm{~m}^{-2}$ (Gierasch et al. 2000) at the lower boundary 
reproduces the large-scale features of Jupiter's uppertropospheric jets. It produces a prograde equatorial jet and an alternating sequence of retrograde and prograde off-equatorial jets, with speeds and widths similar to those on Jupiter (Fig. 1a). The jets maintain their speeds and structure over time in the statistically steady state of the simulation; however, there is some low-frequency variability, most pronounced in low latitudes, with decadal and possibly longer time scales. The equatorial jet is wider and stronger than the off-equatorial jets and resembles Jupiter's equatorial jet, though it does not exhibit Jupiter's local velocity minimum at the equator. As on Jupiter, retrograde off-equatorial jets are weaker than prograde jets, except for the first retrograde jets off the equator; in high latitudes, some retrograde jets are more manifest as local zonal velocity minima than as actual retrograde flow in the upper troposphere. (The retrograde jets are stronger at lower levels, but they are still weaker than the prograde jets within our GCM domain; see Fig. 4 below.) Prograde off-equatorial jets are sharper than retrograde jets, consistent with them being barotropically more stable (Rhines 1994). The meridional gradient of absolute vorticity in the upper troposphere is generally positive but, particularly in shorter-term averages, reverses in the sharpest retrograde jets, where it can approach $-\beta / 2$ - similar to, but of smaller magnitude than, the reversed absolute vorticity gradients in Jupiter's retrograde jets (Ingersoll et al. 1981; Read et al. 2006). That Jupiter's retrograde jets violate sufficient conditions for linear barotropic stability in the upper troposphere has been much discussed and is sometimes deemed puzzling (e.g., Ingersoll et al. 2004; Vasavada and Showman 2005). However, as demonstrated here and previously, for example, by Williams (2002) and Kaspi and Flierl (2007) for baroclinic flows and by Marston et al. (2008) for barotropic flows, it is not necessary that statistically steady states of forced-dissipative flows satisfy barotropic stability conditions for unforced and nondissipative flows.

The speeds and widths of the off-equatorial jets increase with decreasing Rayleigh drag coefficient, similar to the drag dependence of two-dimensional turbulent flows described by Smith et al. (2002) and Danilov and Gurarie (2002). Because the drag coefficient is poorly constrained by data, we empirically chose a value $\left(0.05 \mathrm{day}^{-1}\right.$, with 1 day $\left.=86400 \mathrm{~s}\right)$ that resulted in a good fit to Jupiter's off-equatorial jet speeds and widths. We also experimented with different widths of the region of vanishing Rayleigh drag. Doubling the width of this region such that it extends to $33^{\circ}$ latitude in each hemisphere leads to a prograde equatorial jet that is $\sim 35 \mathrm{~m} \mathrm{~s}^{-1}$ stronger at the equator and whose first zero off the equator is $\sim 5^{\circ}$ farther poleward, giving a closer match to Jupiter's equatorial jet; however, it also leads to the first retrograde jets off the equator being considerably stronger and wider than those on Jupiter. Nonetheless, it is clear that the width of the region of vanishing drag does not alone control the width of the prograde equatorial jet. But having a sufficiently wide region of vanishing or very weak drag around the equator is necessary to obtain equatorial superrotation with intrinsic heat fluxes comparable to Jupiter's; if the drag coefficient in the equatorial region is similar to that in higher latitudes, considerably stronger intrinsic heat fluxes are necessary to generate equatorial superrotation (see appendix B for details).

Instantaneous flow fields in the upper troposphere provide the first evidence that the equatorial superrotation is indeed a consequence of meridional radiation of convectively generated Rossby waves. Although the imposed intrinsic heat flux at the lower boundary is spatially uniform, fluctuations in the horizontal divergence associated with convective heating fluctuations are primarily confined to an equatorial latitude band and there are typically modulated by large-scale waves; the horizontal flow away from the equator is geostrophic and thus nondivergent to leading order (Fig. 3a). Root-mean-square (rms) fluctuations in the horizontal divergence are maximal at the equator, where they reach $\sim 1 \times 10^{-5} \mathrm{~s}^{-1}$; they decay to half their maximum value at $\sim 4^{\circ}$ latitude, consistent with our scaling estimates (see footnote 1 ). In the vertical, rms fluctuations in the horizontal divergence are maximal in the upper troposphere, below the top of the convective outflows (near and slightly above the level at which they are shown in Fig. 3). Similarly, the Rossby wave source (5) is also primarily confined to an equatorial latitude band (Fig. 3b). In the vertical, rms fluctuations in the Rossby wave source (5) are maximal in the upper troposphere, like the horizontal divergence fluctuations. In the horizontal, they are maximal $\sim 2^{\circ}$ off the equator, where they can be generated by vortex stretching and reach $\sim 1.5 \times 10^{-10} \mathrm{~s}^{-2}$; they decay to half their maximum value at $\sim 7^{\circ}$ latitude, consistent with our scaling estimates (footnote 3 ). While fluctuations in the horizontal divergence and in the Rossby wave source (5) are primarily confined to the equatorial region, the vorticity of the horizontal flow shows eddies at all latitudes (Fig. $3 \mathrm{c})$. The vorticity fluctuations are similar in magnitude to the divergence fluctuations near the equator but are much larger than divergence fluctuations away from the equator-consistent with eddy generation by convective heating fluctuations near the equator and eddy generation by baroclinic instability away from the equator. The vorticity field clearly shows the shear zones between the zonal jets as well as smaller-scale coherent vortices, 


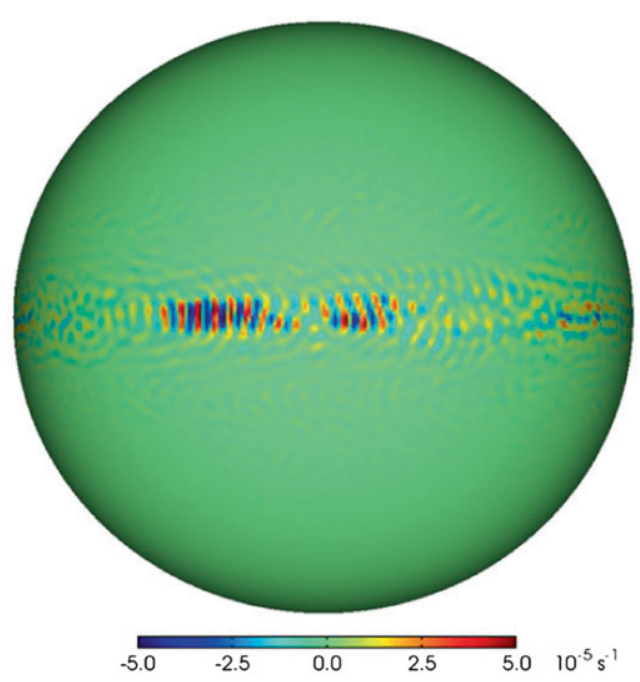

c

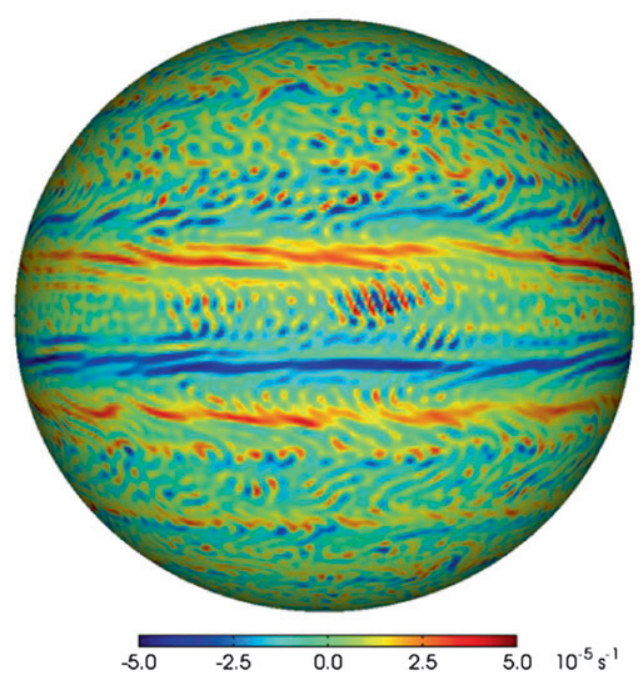

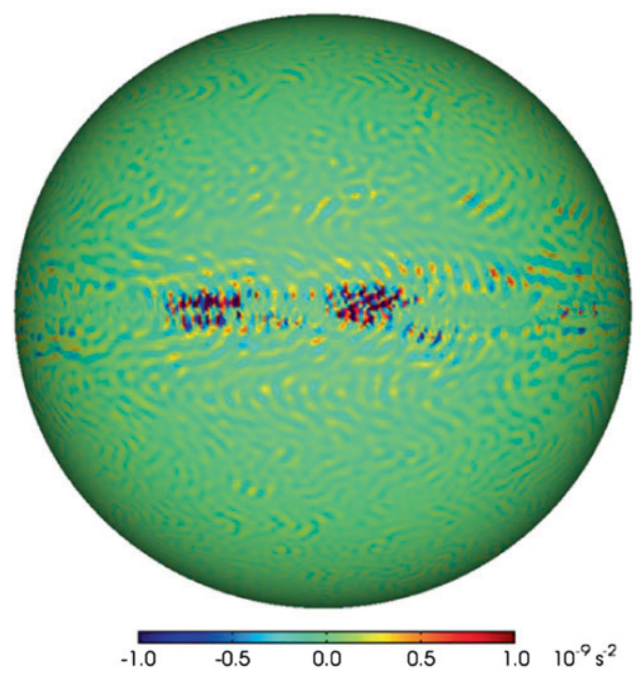

d

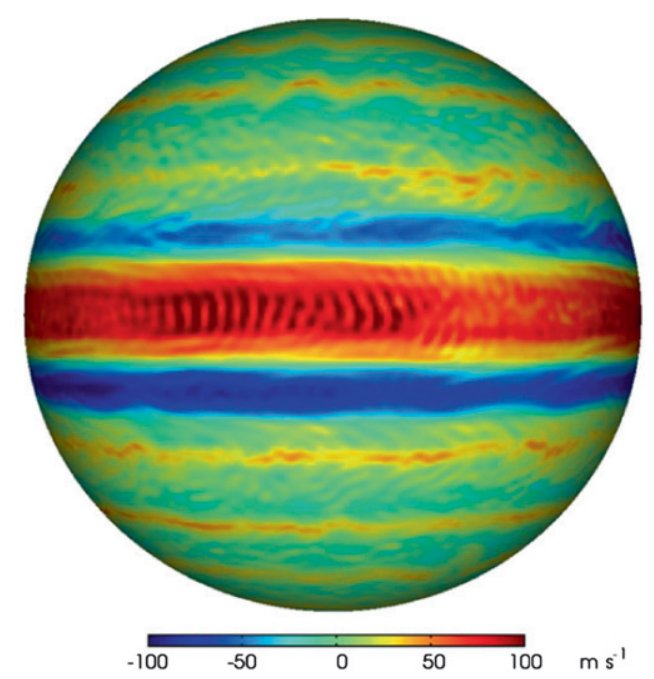

FIG. 3. Flow fields at 0.65 bar at one instant in Jupiter simulation: (a) horizontal divergence, (b) Rossby wave source (5), (c) relative vorticity of horizontal flow, and (d) zonal velocity. The instant shown is within the period for which the mean zonal flow is shown in Fig. 1a.

though no large coherent vortices such as the Great Red Spot. It is possible that large vortices such as the Great Red Spot form spontaneously but would require longer integration times than we can achieve in our simulation, or that deep-atmosphere dynamics not captured in our simulation are important for their formation and stability. That the zonal jets are present and coherent at every instant, not only upon averaging, is most clearly evident in the zonal velocity field, which also shows the equatorial waves recognizable in the other flow fields, as well as undulations of off-equatorial jets (Fig. 3d). Animations (available at www.gps.caltech. edu/ tapio/papers/) show that the equatorial waves, organized into large wave packets, exhibit retrograde phase velocities, consistent with their being Rossby waves. The retrograde tilt of their phase lines away from the equator (Fig. 3d) indicates that they transport angular momentum toward the equator (cf. Peixoto and Oort 1992, chapter 11).

\section{b. Vertical structure and angular momentum fluxes}

The vertical structure of the zonal flow in the simulation indicates preferential baroclinic eddy generation in prograde off-equatorial jets and is consistent with what is known about Jupiter's equatorial jet in lower layers (Fig. 4a). The speed of the prograde equatorial jet increases with depth, for example, at the equator, from 

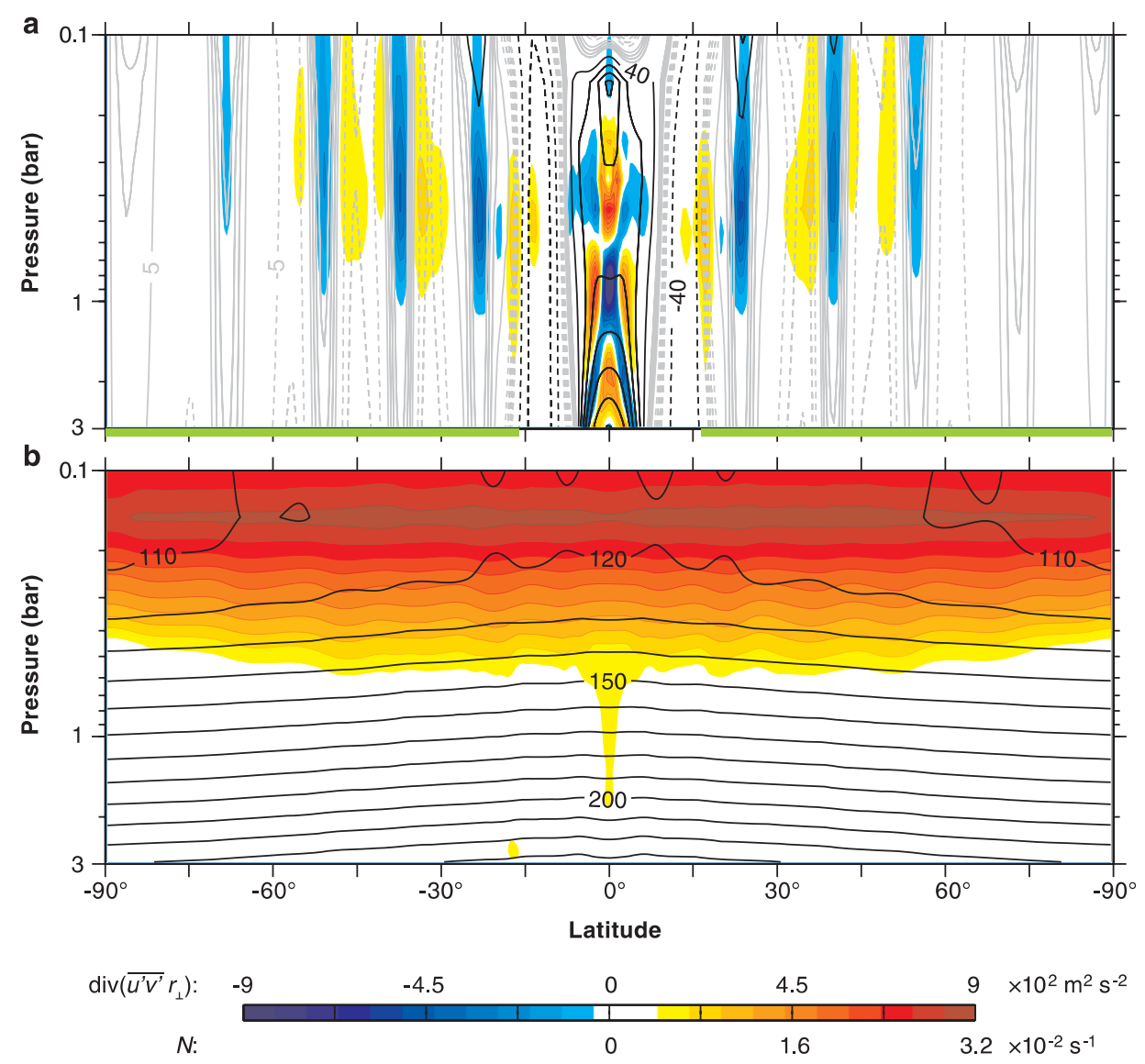

FIG. 4. Flow fields in the latitude-pressure plane in Jupiter simulation. (a) Zonal flow (contours) and divergence div $\left(\overline{u^{\prime} v^{\prime}} r_{\perp}\right)$ of meridional eddy angular momentum fluxes (colors). Gray contours for zonal flow speeds between 5 and $20 \mathrm{~m} \mathrm{~s}^{-1}$, with a contour interval of $5 \mathrm{~m} \mathrm{~s}^{-1}$; black contours for zonal flow speeds of $40 \mathrm{~m} \mathrm{~s}^{-1}$ or above, with a contour interval of $20 \mathrm{~m} \mathrm{~s}^{-1}$. Solid contours for prograde flow and dashed contours for retrograde flow. (b) Temperature (contours, contour interval $10 \mathrm{~K}$ ) and BruntVäisälä frequency $N$ (colors). Shown in this and subsequent figures are zonal and temporal means over the same 1500 days for which the zonal flow at 0.65 bar is shown in Fig. 1a. The green part of the latitude axis marks the latitudes with Rayleigh drag. The graphs are truncated at 0.1 bar at the top, but the uppermost full level of the GCM has a mean pressure of 0.05 bar.

$\sim 80 \mathrm{~m} \mathrm{~s}^{-1}$ between 0.25 and 1 bar to $\sim 160 \mathrm{~m} \mathrm{~s}^{-1}$ at 3 bar. This is similar to the increase in zonal flow speed seen on Jupiter along the Galileo probe descent path at $6.4^{\circ} \mathrm{N}$ (Atkinson et al. 1998), which lies within Jupiter's equatorial jet and so may be comparable to latitudes closer to the equator in our simulation with a narrower equatorial jet. The speed of the off-equatorial zonal flow decreases with depth in prograde jets and increases, with weaker vertical shear, in retrograde jets. For example, the maximum prograde jet speed poleward of $20^{\circ}$ latitude decreases from $30 \mathrm{~m} \mathrm{~s}^{-1}$ at 0.65 bar to $23 \mathrm{~m} \mathrm{~s}^{-1}$ at $3 \mathrm{bar}$, whereas the maximum retrograde jet speed increases from $13 \mathrm{~m} \mathrm{~s}^{-1}$ at 0.65 bar to $15 \mathrm{~m} \mathrm{~s}^{-1}$ at 3 bar. This implies that the prograde off-equatorial jets are baroclinically more unstable than the retrograde jets.
The thermal structure of the atmosphere is in thermal wind balance with the zonal flow, shows the signature of convection penetrating into the upper troposphere, and is consistent with what is known about Jupiter's thermal structure (Fig. 4b). For example, meridional temperature gradients along isobars are poleward (reversed) within the equatorial jet, consistent with thermal wind balance and zonal flow speeds increasing with depth. Away from the equator, they are equatorward in prograde jets but are poleward or close to zero in many retrograde jets, consistent with the opposite signs of the vertical shear in prograde and retrograde jets. The atmosphere is stably stratified above $\sim 0.5$ bar, with a temperature inversion above $\sim 0.2$ bar and with a level of minimum temperature lapse rate (maximum Brunt-Väisälä frequency) at $\sim 0.15$ bar that 
may be identified with the tropopause. These features of the thermal structure are qualitatively and to a large degree quantitatively consistent with the available data for Jupiter's upper troposphere (Simon-Miller et al. 2006). A possible quantitative discrepancy may be that in the layer between 0.1 and 0.5 bar, which contains the thermal emission level on Jupiter as well as in the simulation, pole-equator temperature contrasts along isobars may be a few Kelvin larger in the simulation than on Jupiter. (If it does not result from data and retrieval limitations, the lack of vertical resolution and of representation of hazes in our GCM may account for this discrepancy.) Beneath $\sim 0.5$ bar, the atmosphere in the simulation is nearly neutrally stratified by convection. As a consequence of the nearly neutral stratification, meridional entropy gradients in the convective layer are nearly invariant in the vertical. Close to the equator, large-scale vertical entropy fluxes lead to a weakly stable thermal stratification above $\sim 2$ bar, consistent with Jupiter's stratification along the Galileo probe descent path (Magalhães et al. 2002). The external gravity wave speed implied by the thermal stratification ${ }^{7}$ up to 0.15 bar is $\sim 480 \mathrm{~m} \mathrm{~s}^{-1}$ at the equator, decreases to $\sim 400 \mathrm{~m} \mathrm{~s}^{-1}$ at $5^{\circ}$ latitude, and from there decreases gradually but not monotonically to $\sim 300 \mathrm{~m} \mathrm{~s}^{-1}$ at $80^{\circ}$ latitude-values roughly consistent with the estimated gravity wave speed for Jupiter (Ingersoll and Kanamori 1995).

Consistent with prograde jets being baroclinically more unstable than retrograde jets and with baroclinic eddy generation preferentially in prograde jets, eddies in the simulation transport angular momentum meridionally from retrograde into prograde off-equatorial jets in layers above $\sim 1$ bar (Fig. 4a). Poleward of $20^{\circ}$, the divergence of meridional eddy angular momentum fluxes in retrograde jets reaches up to $170 \mathrm{~m}^{2} \mathrm{~s}^{-2}$ and the convergence in prograde jets up to $470 \mathrm{~m}^{2} \mathrm{~s}^{-2}$, with extremal values attained between 0.2 and 0.6 bar. In the equatorial region, apparently owing to meridional radiation of convectively generated Rossby waves, there is strong convergence of meridional eddy angular momentum fluxes (up to $890 \mathrm{~m}^{2} \mathrm{~s}^{-2}$ ) between 0.6 and

\footnotetext{
${ }^{7}$ We estimated the gravity wave speed as $c=\int_{p_{t}}^{p_{s}} N_{p} d p$, where $p_{s}$ is the pressure at the lower boundary, $p_{t}=0.15$ bar is the upper boundary of the integration, $N_{p}^{2}=-(\bar{\rho} \bar{\theta})^{-1} \overline{\partial_{p} \theta}$ is a static stability measure, and $\rho$ is density (Schneider and Walker 2006). Because the thermal stratification is (nearly) neutral in lower layers, the gravity wave speed depends only weakly on the lower boundary of the integration. However, it decreases as the upper boundary is lowered ( $p_{t}$ is increased) within the stably stratified upper troposphere. For example, with an upper boundary of $p_{t}=0.2$ bar, one obtains a gravity wave speed of $\sim 350 \mathrm{~m} \mathrm{~s}^{-1}$ at the equator.
}

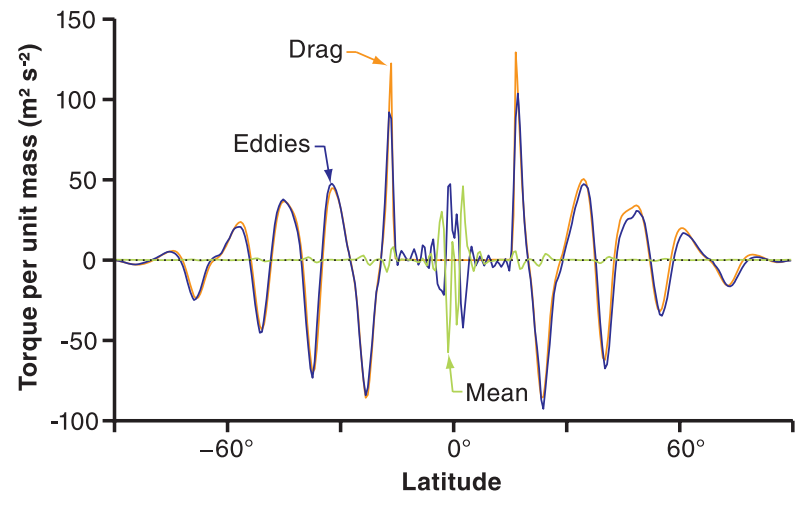

FIG. 5. Zonally, temporally, and vertically averaged angular momentum balance in Jupiter simulation: torque per unit mass owing to divergence of eddy angular momentum fluxes $\left\langle\mathcal{S}_{e}\right\rangle$ (blue), to divergence of mean angular momentum fluxes $\left\langle\mathcal{S}_{m}\right\rangle$ (green), and to drag on the zonal flow $r_{\perp}\langle\overline{\mathcal{D}}\rangle$ (orange).

1.4 bar-below and near the transition from the weakly or neutrally stratified lower layers to the more stably stratified upper layers. There is weaker divergence (up to $660 \mathrm{~m}^{2} \mathrm{~s}^{-2}$ ) at higher and lower levels, as well as immediately off the equator between 0.6 and 2 bar.

The angular momentum balance is closed in the manner discussed in section 4. Away from the equator, in the region with Rayleigh drag, the Rossby number Ro $=U /|f L|$ is small, and mean meridional circulations link divergences and convergences of eddy angular momentum fluxes in the upper troposphere to the drag at the lower boundary. The mean meridional circulations satisfy the local angular momentum balance (14) in the upper troposphere and extend downward along vertical lines (planetary angular momentum contours in the thinshell approximation); they form thin-shell analogs of the circulations sketched in Fig. 2. (In fact, we produced Fig. 2 by projecting winds and mean meridional circulations from a thin-shell GCM simulation into a thick spherical shell.) Although the eddy angular momentum fluxes are confined to a relatively thin layer, the jets extend to the lower boundary, with the signs and zeros of the zonal flow projecting downward approximately along vertical lines (Fig. 4a). This allows the drag on the zonal flow to balance any convergence or divergence of eddy angular momentum fluxes in the vertical average, such that the vertically averaged angular momentum balance in the limit of small Rossby number, $\left\langle\mathcal{S}_{e}\right\rangle \approx r_{\perp}\langle\overline{\mathcal{D}}\rangle$, is approximately satisfied (Fig. 5). In the region of vanishing Rayleigh drag, the vertically averaged angular momentum balance $\left\langle S_{e}+S_{m}\right\rangle \approx 0$ is satisfied by partially compensating divergences and convergences of meridional eddy angular momentum fluxes in different layers (Fig. 4a), as well as vertical eddy fluxes and mean 
meridional circulations redistributing angular momentum between layers and between latitude bands within the equatorial region (Fig. 5). The small residual in the zonally, temporally, and vertically averaged angular momentum balance (Fig. 5) is indicative of sampling variability and is associated with low-frequency variability in the mean zonal flow.

The correlation between the convergence of eddy angular momentum fluxes and the mean flow evident in Figs. 4 and 5 implies conversion of eddy kinetic energy to mean flow kinetic energy. Above 1 bar, the mean conversion rate from eddy to mean flow kinetic energy per unit of mass ${ }^{8}$ is $\sim 2 \times 10^{-5} \mathrm{~W} \mathrm{~kg}^{-1}$. This conversion rate and the eddy angular momentum fluxes themselves are of magnitudes similar to those inferred for Jupiter's upper troposphere by Ingersoll et al. (1981) and Salyk et al. (2006). However, unlike what was assumed in those studies, the eddy angular momentum fluxes in our simulation have a baroclinic structure and are confined to a relatively thin layer in the upper troposphere. As a consequence, the total conversion rate from eddy to mean flow kinetic energy in our simulation is only $0.08 \mathrm{~W} \mathrm{~m}^{-2}$, that is, only $0.5 \%$ of the energy uptake by the atmosphere $\left(14.0 \mathrm{~W} \mathrm{~m}^{-2}\right)$ - an order of magnitude smaller than suggested by Ingersoll et al. (1981) and Salyk et al. (2006). It is also only a fraction (19\%) of the generation rate of eddy kinetic energy by conversion from eddy potential energy $\left(0.41 \mathrm{~W} \mathrm{~m}^{-2}\right)$. The remainder of the eddy kinetic energy generation is balanced by dissipation (by the Rayleigh drag and by the hyperdiffusion representing subgrid-scale processes), similarly as in Earth's atmosphere (cf. Peixoto and Oort 1992, chapter 14).

\section{c. Eddy scales and turbulence characteristics}

Eddies in the simulation exhibit a broad range of length scales, with the dominant scales depending on latitude and on whether zonal or meridional velocity fluctuations are considered. The vertically averaged zonal spectrum of the zonal eddy velocity variance $\overline{u^{\prime 2}}$ exhibits largest power over a broad range of relatively small zonal wavenumbers $m \lesssim 10$ (Fig. 6a). The zonal eddy velocity variance is maximal in the equatorial region, where it peaks at $m=4$ and retains substantial power at yet smaller wavenumbers (see the equatorial waves and wave packets in the instantaneous zonal

\footnotetext{
${ }^{8}$ We calculated energy conversion rates following Lorenz (1955). The conversion rate from eddy to mean flow kinetic energy includes the (small) contributions owing to vertical eddy fluxes of angular momentum and other terms.
}

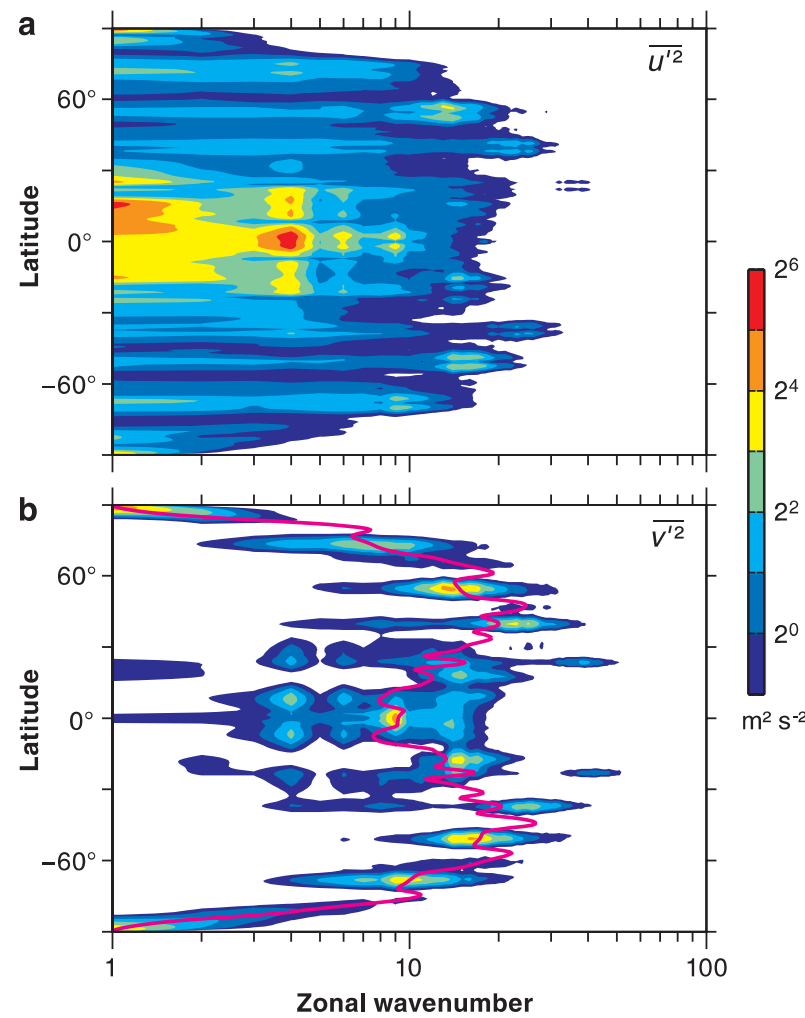

FIG. 6. Mass-weighted vertical mean of zonal spectra of eddy velocity variances: (a) zonal velocity variance $\overline{u^{\prime 2}}$ and (b) meridional velocity variance $\overline{v^{\prime 2}}$. The velocity variance contouring is logarithmic. The magenta line in (b) marks the energy-containing zonal wavenumber $m_{e}(\phi)$, defined as the first negative moment of the zonal spectrum of the meridional eddy velocity variance.

velocity field in Fig. 3d). In contrast, the analogous spectrum of the meridional eddy velocity variance $\overline{v^{\prime 2}}$ has relatively well-defined maxima at larger zonal wavenumbers $m \geqslant 10$, except in polar latitudes, where the maxima are at smaller wavenumbers (Fig. 6b). At wavenumbers $m \geqslant 10$ away from and $m \geqslant 5$ near the poles, zonal and meridional eddy velocity variances are of similar magnitude, indicating approximate isotropy of the eddies. At smaller wavenumbers, the zonal exceeds the meridional eddy velocity variance, indicating that the zonal variance is associated with anisotropic, predominantly zonal flow structures (Boer and Shepherd 1983; Shepherd 1987a). This large-scale zonal variance is likely associated with variations in the zonal jets themselves, for example, with low-frequency undulations. Therefore, the meridional eddy velocity variance is more suitable for defining an energy-containing turbulent eddy scale.

The energy-containing zonal wavenumber $m_{e}(\phi)$, defined as the first negative moment of the vertically averaged zonal spectrum of the meridional eddy 
velocity variance, ${ }^{9}$ closely follows the maximum of the zonal spectrum (Fig. 6b). It increases from $\sim 9$ near the equator to $\sim 25$ near $40^{\circ}$ latitude and then decreases toward the poles. The associated zonal length scale $2 \pi a(\cos \phi) / m_{e}$ decreases from $\sim 50000 \mathrm{~km}$ near the equator to $\sim 18000 \mathrm{~km}$ near $40^{\circ}$ latitude but then varies only weakly, between $\sim 10000$ and $\sim 20000 \mathrm{~km}$, in higher latitudes. Poleward of $\sim 30^{\circ}$ latitude, this zonal length scale is similar to the meridional separation scale between the off-equatorial jets (Fig. 1a), which suggests that the length scale of approximately isotropic eddies controls the jet spacing there.

The change in the energy-containing zonal wavenumber and length scale between equatorial and offequatorial regions marks a transition between different dynamical regimes:

- In the equatorial region, the energy-containing wavenumber $(\sim 9)$ is approximately equal to the wavenumber corresponding to the equatorial Rossby radius $L_{e}=(c / \beta)^{1 / 2}$ (Fig. 7). ${ }^{10}$ Thus, it is approximately equal to the wavenumber of long equatorial Rossby waves. Consistently, the zonal phase velocity spectrum (Randel and Held 1991) of the meridional eddy velocity variance exhibits maximum power at retrograde phase velocities between -50 and $-100 \mathrm{~m} \mathrm{~s}^{-1}$, and the analogous spectrum of the meridional eddy flux of angular momentum peaks at similar phase velocities. ${ }^{11}$ This is in agreement with theory: long equatorial Rossby waves are expected to have retrograde phase velocities of about $-c / 3 \approx-150 \mathrm{~m} \mathrm{~s}^{-1}$ relative to the mean zonal flow (Matsuno 1966; Gill 1982, chapter 11.8).

- Away from the equator, the energy-containing wavenumber is approximately equal to the wavenumber corresponding to the extratropical Rossby

\footnotetext{
${ }^{9}$ That is, given the vertically averaged meridional velocity variance spectrum $E_{v}(m, \varphi)$, the energy-containing wavenumber is defined as the integral scale

$$
m_{e}(\phi)=\left[\frac{\sum_{m \geq 1} m^{-1} E_{v}(m, \phi)}{\sum_{m \geq 1} E_{v}(m, \phi)}\right]^{-1} .
$$

This integral scale more closely follows the maximum of the zonal spectrum than do other low-order moments of the spectrum.

${ }^{10}$ For an approximately isotropic equatorial Rossby wave with meridional decay scale $L_{e}$, we assume that the zonal wavelength is $2 \pi L_{e}$, and hence the zonal wavenumber is $2 \pi a \cos \phi /$ $\left(2 \pi L_{e}\right)=a(\cos \phi) / L_{e}$ (cf. Matsuno 1966). Other zonal wavenumbers in what follows, in which $O(1)$ constants in the relevant length scales $L$ can be adjusted in any case, are defined analogously as $a(\cos \phi) / L$, that is, without factors of $2 \pi$.

${ }^{11}$ Long waves with retrograde phase velocities are also clearly evident in the zonal velocity animations available at www.gps. caltech.edu/ $\sim$ tapio/papers/.
}

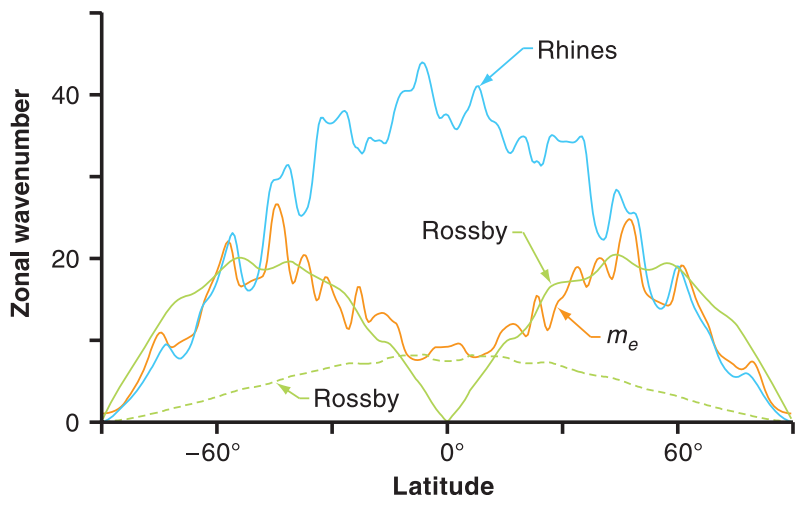

FIG. 7. Zonal wavenumbers in Jupiter simulation. Orange: energy-containing wavenumber $m_{e}$. Light blue: Rhines wavenumber $a(\cos \phi) / L_{\beta}$ with $L_{\beta}=\gamma_{\beta} \mathrm{EKE}_{\mathrm{bt}}^{1 / 4} / \beta^{1 / 2}$, barotropic eddy kinetic energy per unit mass EKE $E_{\mathrm{bt}}$, and empirical constant $\gamma_{\beta}=$ 1.6. Green dashed: equatorial Rossby wavenumber $a(\cos \phi) / L_{e}$ with $L_{e}=(c / \beta)^{1 / 2}$. Green solid: extratropical Rossby wavenumber $a(\cos \phi) / L_{x}$ with $L_{x}=\gamma_{x} c /|f|$ and empirical constant $\gamma_{x}=1.8$. The gravity wave speed $c$ entering the Rossby wavenumbers is estimated from the thermal stratification between the lower boundary and 0.15 bar (see footnote 7 ).

radius $L_{x}=\gamma_{x} c /|f|$, where we fixed the empirical constant at $\gamma_{x}=1.8$ (Fig. 7). Thus, it scales approximately with the expected wavenumber of the baroclinically most unstable linear waves. In middle and high latitudes, the energy-containing wavenumber is also closely approximated by the wavenumber corresponding to the Rhines scale $L_{\beta}=\gamma_{\beta} \mathrm{EKE}_{\mathrm{bt}}{ }^{1 / 4} / \beta^{1 / 2}$, obtained by combining the barotropic eddy kinetic energy per unit mass $\mathrm{EKE}_{\mathrm{bt}}$ (the eddy kinetic energy of the vertically averaged flow) with $\beta$ (Rhines 1975, 1994); we fixed the empirical constant at $\gamma_{\beta}=1.6$ as in O'Gorman and Schneider (2008). The Rhines wavenumber even captures the variations in the energycontaining wavenumber between retrograde and prograde jets; however, equatorward of $\sim 45^{\circ}$ latitude, the Rhines wavenumber exceeds the energy-containing wavenumber (Fig. 7).

These results are consistent with convectively generated equatorial Rossby waves dominating the meridional eddy velocity variance in the equatorial region and baroclinically generated eddies dominating away from the equator.

The coincidence of the energy-containing scale with the Rossby radius and the jet separation scale away from the equator indicates that the off-equatorial jets form without an inverse cascade of barotropic eddy kinetic energy (or at least without an inverse energy cascade over an extended inertial range). The fact that the energy-containing scale is larger than the Rhines scale equatorward of $\sim 45^{\circ}$ latitude also points to the 
absence of an inverse energy cascade; the coincidence of the energy-containing scale with the Rhines scale at higher latitudes is no evidence to the contrary (Schneider and Walker 2006). Indeed, consistent with the absence of an inverse energy cascade, the global barotropic eddy kinetic energy spectrum as a function of spherical wavenumber $n$ does not show the flattening to an $n^{-5 / 3}$ power law at large scales that would be expected if an inverse energy cascade were occurring (Fig. $8 a)$. The nonlinear spectral flux of total kinetic energy shows upscale transfer of kinetic energy over a range of wavenumbers between $n \sim 10$ and 100 (Fig. 8b), but it is dominated by interactions that involve the zonal-mean flow (i.e., the $m=0$ component; dashed-dotted line in Fig. 8b). Interactions that involve only eddies (i.e., only zonal wavenumbers $m>0$; dashed line in Fig. 8b) and that could give rise to an inverse energy cascade are much weaker, and they do not exhibit an extended wavenumber range in which their nonlinear spectral flux is constant, as would be expected in an inertial range. It is evident that eddy-mean flow interactions, in addition to or in place of nonlinear eddy-eddy interactions, are fundamental for the formation of the offequatorial jets (O'Gorman and Schneider 2007, 2008). This situation is similar to that in Earth's atmosphere, in which, likewise, eddy-mean flow interactions are more important than nonlinear eddy-eddy interactions for the upscale transfer of kinetic energy, and there is no extended inertial range (Shepherd 1987a,b).

\section{Control simulations}

To demonstrate that in our GCM, differential radiative heating is indeed responsible for off-equatorial jets and intrinsic convective heat fluxes for equatorial superrotation, we performed one simulation without differential radiative heating and one without intrinsic heat fluxes. In the simulation without differential radiative heating, an intrinsic heat flux of $5.7 \mathrm{~W} \mathrm{~m}^{-2}$ is imposed at the lower boundary, as in the Jupiter simulation, but insolation at the top of the atmosphere is uniform and equal to the global-mean insolation in the Jupiter simulation. In the simulation without intrinsic heat fluxes, insolation at the top of the atmosphere varies with the cosine of latitude, as in the Jupiter simulation, but no heat flux is imposed at the lower boundary. Other parameters in these control simulations are identical to those in the Jupiter simulation.

With the intrinsic heat flux but without differential radiative heating, a prograde equatorial jet forms, flanked by two retrograde jets; however, there are no offequatorial jets (Fig. 1b). With differential radiative heating but without intrinsic heat fluxes, off-equatorial
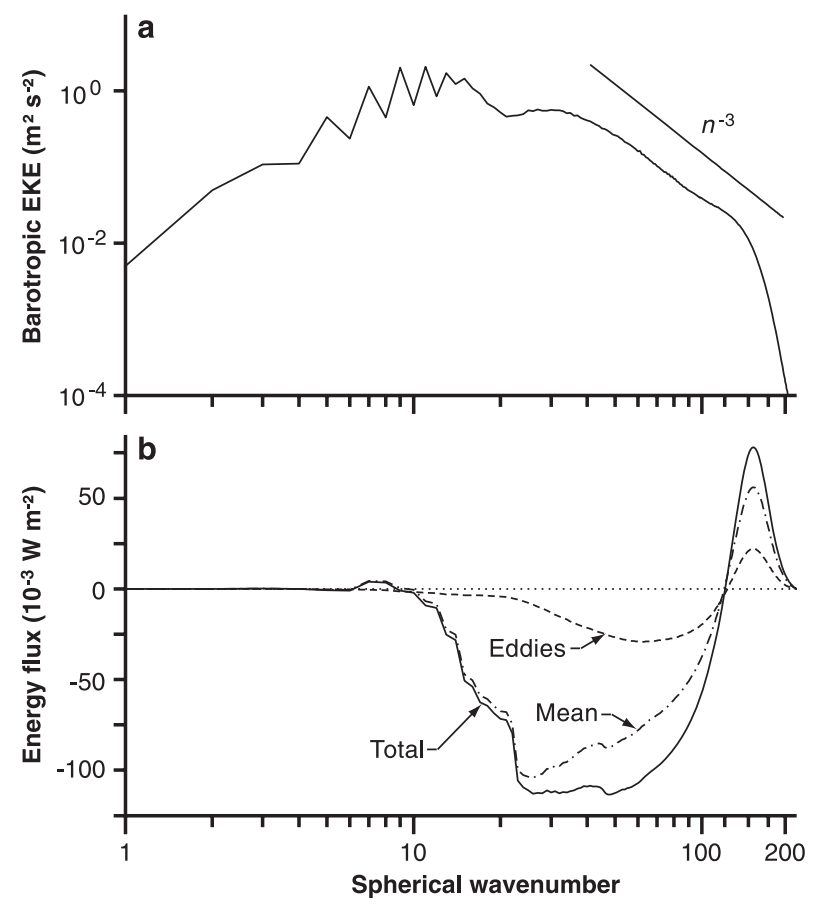

FIG. 8. Eddy kinetic energy spectrum and spectral energy fluxes as a function of spherical wavenumber. (a) Spectrum of barotropic eddy kinetic energy (eddy kinetic energy of vertically averaged flow), with an $n^{-3}$ power law in spherical wavenumber $n$ for comparison. (b) Nonlinear spectral flux of total (globally integrated) kinetic energy (solid), decomposed into components involving interactions with the zonal mean (dashed-dotted) and involving only eddy-eddy interactions (dashed). Positive fluxes indicate downscale and negative fluxes upscale energy transfer. The spectrum and spectral fluxes are calculated following Boer (1983) and Shepherd (1987a).

jets form with similar speeds and widths as in the Jupiter simulation; however, the equatorial zonal flow is retrograde (Fig. 1b).

The jets that do form in either case again extend to the lower boundary, and the thermal structure is in thermal wind balance with the zonal flow. In the simulation without differential radiative heating (Fig. 9, left column), as in the Jupiter simulation, the thermal structure shows the signature of convection penetrating into the upper troposphere, with stable stratification above $\sim 0.5$ bar and nearly neutral stratification beneath. The speed of the prograde equatorial jet again increases with depth, albeit more weakly than in the Jupiter simulation (cf. Fig. 4a). The speed of the equatorial jet is smaller than in the Jupiter simulation, probably because the control simulation lacks disturbances originating outside the equatorial region, which may trigger convective heating fluctuations and/or may interact with the equatorial mean zonal flow in the Jupiter simulation. The atmosphere outside the equatorial region is essentially 


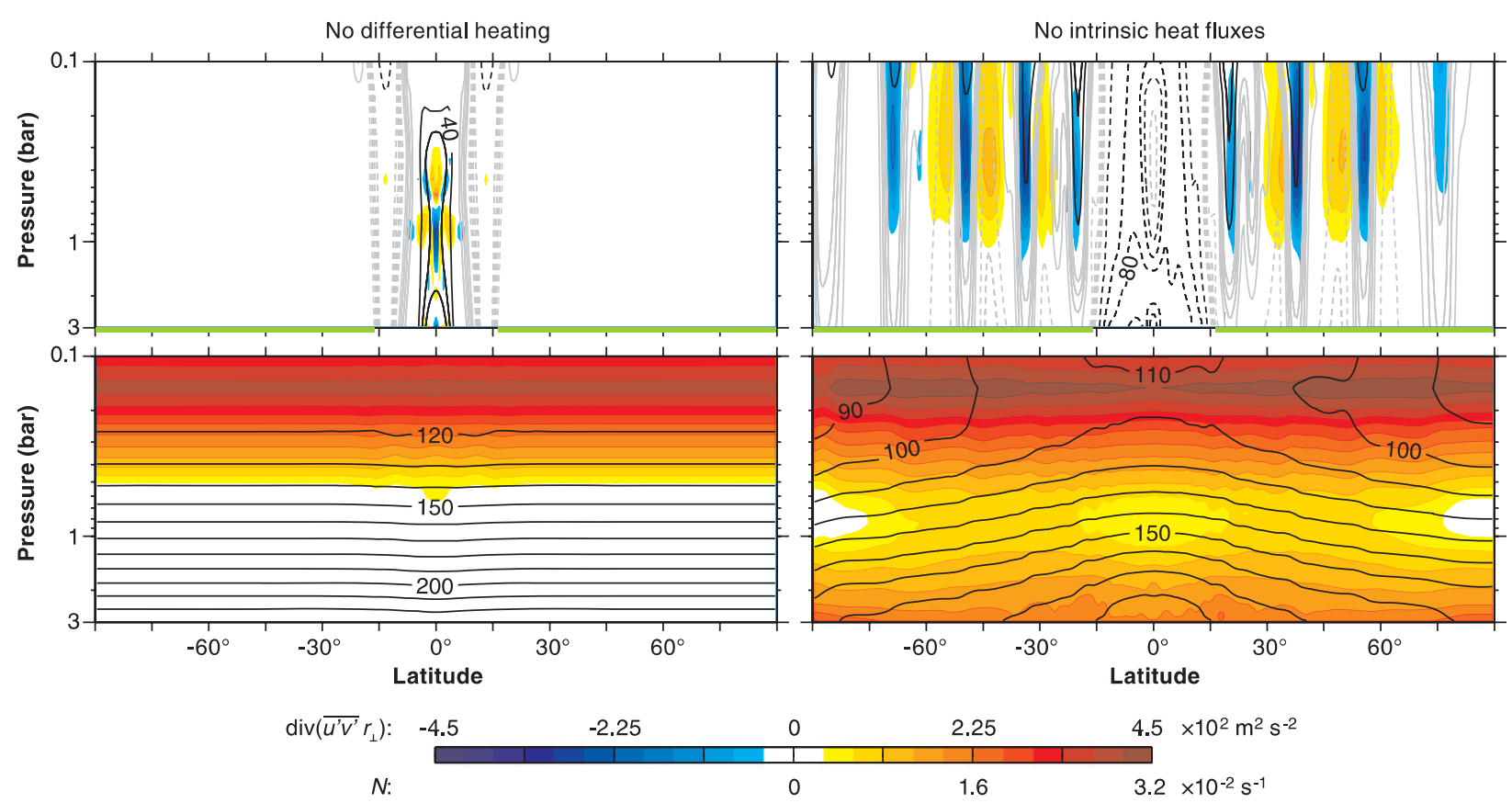

FIG. 9. Flow fields in the latitude-pressure plane in control simulations. (top row) Zonal flow (contours) and $\operatorname{divergence} \operatorname{div}\left(\overline{u^{\prime} v^{\prime}} r_{\perp}\right)$ of meridional eddy angular momentum fluxes (colors). (bottom row) Temperature (contours) and Brunt-Väisälä frequency $N$ (colors). Contour intervals and plotting conventions are as in Fig. 4, except that the contour interval for the angular momentum flux divergence is halved. (left column) Simulation with intrinsic heat fluxes but with uniform insolation at the top of the atmosphere. (right column) Simulation with differential insolation but without intrinsic heat fluxes. Shown are zonal and temporal means over the same 900 simulated days for which the zonal flows at 0.65 bar are shown in Fig. 1 b.

in radiative-convective equilibrium. This illustrates that convection does not necessarily generate kinetic energy on large scales, although the convective heating in the simulation fluctuates at all latitudes (cf. Emanuel et al. 1994). In the equatorial region, the weakness of horizontal temperature gradients implies that convective heating fluctuations cannot generate substantial temperature fluctuations but instead, if externally forced, immediately generate large-scale horizontal divergence and vorticity fluctuations and thus large-scale kinetic energy (Sobel et al. 2001). But outside the equatorial region, convective heating fluctuations can generate local temperature fluctuations, which can decay through radiative processes and dispersion by small-scale flows that eventually dissipate, without generating substantial large-scale kinetic energy. The assumption commonly made in shallow-water models of Jupiter's upper atmosphere-that convective heating fluctuations even away from the equator directly generate large-scale kinetic energy - is in need of justification.

In the simulation without intrinsic heat fluxes (Fig. 9, right column), most of the atmosphere is stably stratified, except for a nearly neutral layer around $\sim 0.8$ bar in high latitudes. The flow throughout the equatorial region is retrograde. The speed of the off-equatorial zonal flow decreases with depth in prograde jets and increases, with weaker vertical shear, in retrograde jets, similarly as in the Jupiter simulation (cf. Fig. 4a). The prograde vertical shear of the zonal flow and, with it, equatorward temperature gradients along isobars are generally larger in the simulation without intrinsic heat fluxes, apparently because the baroclinicity of the stably stratified atmosphere in the control simulation is smaller than that of the atmosphere in the Jupiter simulation with a nearly neutrally stratified layer; the smaller baroclinicity implies weaker meridional eddy entropy fluxes and thus larger meridional temperature gradients along isobars (cf. Schneider and Walker 2008). Consistent with preferential eddy generation by baroclinic instability in prograde jets, there is again meridional eddy angular momentum transport from retrograde into prograde off-equatorial jets in layers above $\sim 1$ bar.

The control simulations confirm that in our GCM, both differential radiative heating and intrinsic convective heat fluxes are necessary to reproduce Jupiter's observed jets and thermal structure. The imposed intrinsic heat flux needs to exceed a threshold value so that convection penetrates into the upper troposphere. Calculations of radiative equilibrium states show that with the representation of radiative processes in our 
GCM, the intrinsic heat flux needs to exceed $\sim 2 \mathrm{~W} \mathrm{~m}^{-2}$ for the troposphere to become statically unstable. Indeed, the zonal flow in a simulation in which an intrinsic heat flux of $2 \mathrm{~W} \mathrm{~m}^{-2}$ is imposed is similar to that in the control simulation without intrinsic heat fluxes.

\section{Conclusions and implications}

Based on the theory and simulations and consistent with the available observational data, we propose that baroclinic eddies generated by differential radiative heating are responsible for Jupiter's off-equatorial jets, and that Rossby waves generated by intrinsic convective heat fluxes are responsible for the equatorial superrotation. Mean meridional circulations adjust entropy gradients and the zonal flow in lower layers of Jupiter's atmosphere such that the zonal flow is in thermal wind balance with the entropy gradients, and convergence or divergence of angular momentum fluxes in the upper troposphere and the MHD drag on the zonal flow at depth balance upon averaging over cylinders concentric with the planet's spin axis. As demonstrated by the Jupiter simulation, the resulting view of how the zonal flow and general circulation are generated and maintained is consistent with observed large-scale features of Jupiter's jets and thermal structure, such as the zonal flow and meridional temperature variations in the upper troposphere and the thermal stratification of the upper troposphere and layers beneath. It is also consistent with the observed eddy angular momentum fluxes and with energetic constraints indicating that these fluxes are confined to a relatively thin atmospheric layer. As demonstrated by the control simulations, differential radiative heating alone can account for the off equatorial jets, and intrinsic convective heat fluxes can account for the prograde equatorial jet. However, intrinsic convective heat fluxes alone do not necessarily lead to formation of off-equatorial jets.

The theory and simulations predict aspects of the general circulation that have not been observed but that are or soon will be observable. For example, the transition in energy-containing eddy scale between the equatorial region and regions away from the equator (Fig. 7), pointing to different mechanisms of eddy generation, should be observable by tracking cloud features. And we predict that the measurements of NASA's upcoming Juno mission to Jupiter will be consistent with zonal jets that extend deeply into the atmosphere at all latitudes, away from the equator up to depths at which the MHD drag acts. As already observed in the upper troposphere, we expect that also at lower layers the speeds of the prograde offequatorial jets decrease with depth and that there are associated equatorward temperature gradients along isobars: qualitatively as in Figs. 4a,b, but likely not quantitatively so because the jets are expected to extend to much greater depth than in our simulations and thus will likely have weaker shear. Depending on the strength of the MHD drag and on the depth at which it acts, the speeds of the retrograde off-equatorial jets may decrease or increase with depth, with weaker shear than the prograde jets, and with associated poleward (reversed) or weaker equatorward temperature gradients. If the shear of the zonal flow can be inferred from measurements, it can be used together with observations of the eddy angular momentum transport and with the implied transfer of kinetic energy from eddies to the mean flow to constrain the strength and depth of the MHD drag and thus to elucidate dynamics of the deep atmosphere that are not amenable to direct measurement.

The proposed mechanisms are generic and likely act in the atmospheres of all giant planets. They suggest, for example, that the reason that Saturn's prograde equatorial jet is wider and stronger than Jupiter's may be that Saturn's tropospheric gravity wave speed and equatorial Rossby radius are greater. The greater depth at which MHD drag on Saturn is estimated to act implies a wider region over which there is effectively no drag on the zonal flow (Liu et al. 2008), thus making a wider equatorial jet possible. The proposed mechanisms also suggest that the reason Uranus and Neptune do not exhibit equatorial superrotation may be that their intrinsic heat fluxes are not sufficiently strong to lead to convection penetrating into the upper troposphere.

Acknowledgments. This work was supported by a David and Lucile Packard Fellowship. The GCM is based on the Flexible Modeling System of the Geophysical Fluid Dynamics Laboratory; the simulations were performed on Caltech's Division of Geological and Planetary Sciences Dell cluster. We thank Isaac Held, Andrew Ingersoll, Yohai Kaspi, Paul O'Gorman, Adam Sobel, David Stevenson, and Paul Wennberg for comments on drafts of this paper and Paul O'Gorman for providing code for the calculation of spectral energy fluxes.

\section{APPENDIX A}

\section{Average Angular Momentum Balance and Rossby Numbers}

An average angular momentum balance follows from the balance equation 


$$
\partial_{t}(\rho M)+\nabla \cdot(\rho \mathbf{u} M)=-\partial_{\lambda} p+r_{\perp} \rho \mathcal{D}
$$

for the angular momentum per unit mass about the planet's spin axis, $M$, with longitude (azimuth) $\lambda$, threedimensional velocity vector $\mathbf{u}$, distance to the planet's spin axis $r_{\perp}$, and drag force per unit mass on the zonal flow $\mathcal{D}$. The angular momentum per unit mass $M=M_{\Omega}+$ $M_{u}$ consists of a planetary component $M_{\Omega}=\Omega r_{\perp}^{2}$ owing to the planetary rotation and a relative component $M_{u}=$ $u r_{\perp}$ owing to the relative zonal velocity $u$ of the atmosphere (e.g., Peixoto and Oort 1992, chapter 11). In this form, the angular momentum balance (A1) is exact (up to neglected viscous stresses) and holds irrespective of the constitutive laws of the atmosphere. Averaging it temporally and zonally (over azimuth and at constant $\left.r_{\perp}\right)$ gives, in a statistically steady state,

$$
\nabla \cdot\left(\overline{\rho \mathbf{u} M_{u}}+\overline{\rho \mathbf{u}} M_{\Omega}\right)=r_{\perp} \overline{\rho \mathcal{D}},
$$

where the overbar denotes a zonal and temporal mean. This is the general, coordinate-independent form of the zonally and temporally averaged angular momentum balance (10) in pressure coordinates in section 3. If one additionally averages (A2) along surfaces of constant $M_{\Omega}$ (i.e., over lines of constant $r_{\perp}$ in the meridional plane) and uses that in a statistically steady state, there can be no net mass flux across such fixed surfaces; it follows that only the zonally and temporally averaged relative angular momentum fluxes $\overline{\rho \mathbf{u} M_{u}}$ across an $M_{\Omega}$ surface contribute to any net convergence or divergence of angular momentum fluxes and to any net acceleration or deceleration of the zonal flow averaged over the $M_{\Omega}$ surface. This acceleration or deceleration must be balanced by drag somewhere on the $M_{\Omega}$ surface; that is,

$$
\nabla \cdot\left\{\overline{\rho \mathbf{u} M_{u}}\right\}=r_{\perp}\{\overline{\rho \mathcal{D}}\},
$$

where $\{\cdot\}$ denotes the mean along lines of constant $r_{\perp}$ in the meridional plane. This is the general, coordinateindependent form of the angular momentum balance (13) in section 4, where the relative angular momentum flux $\overline{\rho \mathbf{u} M_{u}}$ is decomposed into mean and eddy components and the zonal and temporal mean is taken along isobars. In the thin-shell approximation, $r_{\perp}=a \cos \phi$ depends only on latitude $\phi$; the average over $M_{\Omega}$ surfaces is the usual zonal and vertical average (e.g., Vallis 2006). In a deep atmosphere, $r_{\perp}=r \cos \phi$ is the actual distance to the planet's spin axis; the average over $M_{\Omega}$ surfaces is an average along cylinders concentric with the spin axis.

The relative magnitude of relative and planetary angular momentum advection by Eulerian mean flows is indicated by Rossby numbers, with the relevant Rossby number depending on the direction of the flows con- sidered. For meridional flows (i.e., flows along meridians on spheres concentric with the planet's center), the ratio of relative to planetary angular momentum advection scales as $\partial_{y} M_{u} / \partial_{y} M_{\Omega} \sim U /|f L|$ because $\partial_{y} M_{\Omega}=$ $-r_{\perp} f$, where $y$ is the meridional coordinate (arc length) and $L \ll r_{\perp}$ is the meridional length scale of zonal-flow variations; thus, this ratio scales with the usual Rossby number Ro $=U /|f L|$. For cylindrically radial flows (i.e., flows perpendicular to the planet's spin axis), the ratio of relative to planetary angular momentum advection scales as $\partial_{r_{\perp}} M_{u} / \partial_{r_{\perp}} M_{\Omega} \sim U /\left(2 \Omega L_{\perp}\right)$ because $\partial_{r_{\perp}} M_{\Omega}=$ $2 \Omega r_{\perp}$, where $L_{\perp} \ll r_{\perp}$ is the cylindrically radial length scale of zonal-flow variations; thus, this ratio scales with the Rossby number $\mathrm{Ro}_{\perp}=U /\left(2 \Omega L_{\perp}\right)$.

\section{APPENDIX B}

\section{Jupiter GCM}

The GCM is based on the Flexible Modeling System developed at NOAA's Geophysical Fluid Dynamics Laboratory. It uses standard Jupiter parameters (Table B1), except where noted, and integrates the hydrostatic primitive equations for a dry ideal-gas atmosphere in a thin spherical shell, with a stress-free upper boundary at zero pressure and a lower boundary with constant geopotential and with a mean pressure of 3 bar.

\section{a. Discretization and resolution}

The primitive equations in Bourke's (1974) vorticitydivergence form are discretized with the spectral transform method in the horizontal, finite differences in the vertical, and with semi-implicit time differencing (e.g., Durran 1999, chapter 7.6). The horizontal spectral resolution of the GCM is T213 (triangular truncation of the spherical harmonics expansion at wavenumber 213), with $640 \times 320$ (longitude $\times$ latitude) points on the Gaussian transform grid. The vertical coordinate is $\sigma=$ $p / p_{s}$ (pressure $p$ normalized by pressure at lower boundary $p_{s}$ ) and is discretized with 30 equally spaced levels. With this vertical discretization, the density varies by two orders of magnitude from the top to the bottom of the domain.

\section{b. Radiative transfer}

Radiative transfer is represented as that in a homogeneous gray atmosphere, using the two-stream approximation. The top-of-atmosphere (TOA) insolation is imposed as a perpetual equinox with no diurnal cycle,

$$
F_{\mathrm{TOA}}=\frac{F_{0}}{\pi} \cos \phi
$$


TABLE B1. Parameters in Jupiter GCM.

\begin{tabular}{lll}
\hline \hline \multicolumn{1}{c}{ Parameter, symbol } & \multicolumn{1}{c}{ Value } & \multicolumn{1}{c}{ Reference } \\
\hline Planetary radius (at 3 bar), $a$ & $69.86 \times 10^{6} \mathrm{~m}$ & Guillot (1999) \\
Planetary angular velocity, $\Omega$ & $1.7587 \times 10^{-4} \mathrm{~s}$ & Donivan and Carr (1969) \\
Gravitational acceleration, $g$ & $26.0 \mathrm{~m} \mathrm{~s}^{-2}$ & Lodders and Fegley (1998) \\
Specific gas constant, $R$ & $3605.38 \mathrm{~J} \mathrm{~kg}^{-1} \mathrm{~K}^{-1}$ & Lodders and Fegley (1998) \\
Adiabatic exponent, $\kappa$ & $2 / 7$ & \\
Specific heat capacity, $c_{p}=R / \kappa$ & $12619.0 \mathrm{~J} \mathrm{~kg}^{-1} \mathrm{~K}^{-1}$ & Levine et al. (1977) \\
Solar constant, $F_{0}$ & $50.7 \mathrm{~W} \mathrm{~m}^{-2}$ & Gierasch et al. (2000) \\
Intrinsic heat flux & $5.7 \mathrm{~W} \mathrm{~m}^{-2}$ & Hanel et al. (1981) \\
Bond albedo, $r_{\infty}$ & 0.343 & Sromovsky and Fry (2002) \\
Single scattering albedo, $\tilde{\omega}$ & 0.8 &
\end{tabular}

where $F_{0}=50.7 \mathrm{~W} \mathrm{~m}^{-2}$ is the solar constant (Levine et al. 1977).

The solar optical depth $\tau_{s}$ is linear in pressure to represent scattering and absorption by a well-mixed absorber,

$$
\tau_{s}=\tau_{s 0}\left(\frac{p}{p_{0}}\right),
$$

where $\tau_{s 0}$ is the solar optical depth at pressure $p_{0}$. We assume diffuse incidence of solar radiation at TOA. The solar flux $F$ for a semi-infinite scattering and absorbing atmosphere then is (Petty 2006)

$$
F=F_{\mathrm{TOA}}\left(1-r_{\infty}\right) \exp \left(-\Gamma \tau_{s}\right)
$$

where

$$
\Gamma=2 \sqrt{1-\tilde{\omega}} \sqrt{1-\tilde{\omega} \gamma}
$$

and the Bond albedo $r_{\infty}$ can be represented as

$$
r_{\infty}=\frac{\sqrt{1-\tilde{\omega} \gamma}-\sqrt{1-\tilde{\omega}}}{\sqrt{1-\tilde{\omega} \gamma}+\sqrt{1-\tilde{\omega}}} .
$$

Here, $\tilde{\omega}$ is the single-scattering albedo and $\gamma$ the asymmetry factor, which we chose to be $\tilde{\omega}=0.8$ and $\gamma=$ 0.204 to give Jupiter's Bond albedo of $r_{\infty}=0.343$ (Sromovsky and Fry 2002; Hanel et al. 1981). For the solar optical depth, we chose $\tau_{s 0}=3$ at $p_{0}=3$ bar to give a radiative flux $F$ qualitatively consistent with measurements along the Galileo probe descent path in Jupiter, except in the region of hazes in the upper atmosphere (Sromovsky et al. 1998). With these parameters, the solar radiative flux at the lower boundary of the GCM is less than 9\% of the incident flux at the top of the atmosphere.

The thermal optical depth $\tau_{l}$ is quadratic in pressure to represent collision-induced absorption,

$$
\tau_{l}=\tau_{l 0}\left(\frac{p}{p_{0}}\right)^{2}
$$

where $\tau_{l 0}$ is the thermal optical depth at pressure $p_{0}$. We chose $\tau_{l 0}=80$ at $p_{0}=3$ bar, again to give radiative fluxes qualitatively consistent with measurements along the Galileo probe descent path and to give a thermal emission level (where $\tau_{l} \sim 1$ ) in the vicinity of $\sim 0.4$ bar (Ingersoll 1990; Sromovsky et al. 1998).

At the lower boundary, energy conservation is imposed: at each grid point, the upward thermal radiative flux is set equal to the sum of the downward solar and thermal radiative fluxes.

\section{c. Intrinsic heat flux}

A spatially uniform and temporally constant heat flux is deposited in the lowest layer of the GCM to mimic Jupiter's intrinsic heat flux. We use a heat flux of $5.7 \mathrm{~W} \mathrm{~m}^{-2}$ (Gierasch et al. 2000), except in the control simulation without intrinsic heat fluxes.

\section{d. Convection scheme}

A quasi-equilibrium convection scheme represents (dry) convection. It relaxes temperature profiles toward a convective profile with adiabatic lapse rate $g / c_{p} \approx$ $2.1 \mathrm{~K} \mathrm{~km}^{-1}$ whenever an air parcel lifted adiabatically from the lowest model level has positive convective available potential energy (Schneider and Walker 2006). The convective relaxation time is $6 \mathrm{~h}$, chosen as roughly the time it takes a gravity wave with speed $c \approx$ $450 \mathrm{~m} \mathrm{~s}^{-1}$ to traverse the equatorial Rossby radius $(c / \beta)^{1 / 2} \approx 9500 \mathrm{~km}$. The convection scheme conserves enthalpy integrated over atmospheric columns; it does not transport momentum. It can be viewed as a dry limit of the Betts-Miller convection scheme (Betts 1986; Betts and Miller 1986).

\section{e. Drag at lower boundary}

In Jupiter's atmosphere, the conductivity of hydrogen increases with depth, making a continuous transition from very low values in the outer atmosphere to a constant value reached at the depth at which hydrogen 
becomes metallic, which occurs at $\sim 1.4$ Mbar or $\sim 0.84$ Jupiter radii (Nellis et al. 1996; Liu et al. 2008). Where the atmosphere is electrically conducting, the interaction of the magnetic field with the flow leads to Ohmic dissipation and retards the flow (Liu et al. 2008).

As an idealized representation of effects of this MHD drag on the flow in the outer atmosphere, we use Rayleigh drag near the GCM's lower boundary in the horizontal momentum equations,

$$
\partial_{t} \mathbf{v}+\cdots=-k(\phi, \sigma) \mathbf{v} .
$$

As in Held and Suarez (1994), the Rayleigh drag coefficient $k(\phi, \sigma)$ decreases linearly in $\sigma$ from its value $k_{0}(\phi)$ at the lower boundary at $\sigma=1$ to zero at $\sigma_{b}=0.8$,

$$
k(\phi, \sigma)=k_{0}(\phi) \max \left(0, \frac{\sigma-\sigma_{b}}{1-\sigma_{b}}\right) .
$$

To represent in the thin-shell approximation the downward projection along cylinders in a deep atmosphere, we use a drag coefficient $k_{0}(\phi)$ that is constant $\left(k_{0}=\right.$ $0.05 \mathrm{day}^{-1}$ ) poleward of $16.3^{\circ}$ latitude, corresponding to 0.96 planetary radii in a projection onto the equatorial plane, and that exponentially decreases to zero at lower latitudes (Fig. B1).

The kinetic energy dissipated by the Rayleigh drag is returned to the flow as heat to conserve energy.

\section{f. Subgrid-scale dissipation}

Horizontal hyperdiffusion in the vorticity, divergence, and temperature equations acts at all levels and is the only frictional process above the layer with Rayleigh drag $(\sigma \leq 0.8)$. The hyperdiffusion is represented by an exponential cutoff filter (Smith et al. 2002), with a damping time scale of $2 \mathrm{~h}$ at the smallest resolved scale and with no damping for spherical wavenumbers smaller than 100 .

\section{g. Simulations}

The simulations were spun up from radiative-convective equilibrium temperature profiles with no flow, with small random perturbations in temperature and vorticity to break the axisymmetry of the initial state. All simulations were first spun up at T85 horizontal resolution for 10000 simulated (Earth) days. The end states of the T85 simulations were used as initial states of T213 simulations, which were spun up for at least 18000 additional days. Over the first 10000 days of the T213 simulations, we experimented with Rayleigh drag parameters near the lower boundary to obtain a good fit to Jupiter's observed zonal flow. The Rayleigh drag parameters were held fixed at the values stated above

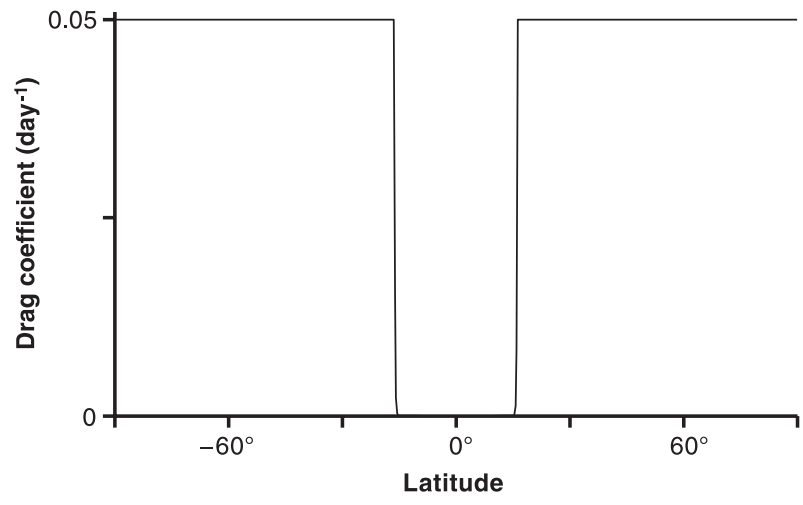

FIG. B1. Rayleigh drag coefficient $k_{0}(\phi)$ at the GCM's lower boundary.

for at least 8000 days of spinup of the T213 simulations, until statistically steady states were reached.

In the statistically steady states, the global-mean outgoing thermal radiative flux is within $\lesssim 0.02 \mathrm{~W} \mathrm{~m}^{-2}$ of the sum of the global-mean absorbed solar radiative flux and the imposed intrinsic heat flux. The vertically integrated Rayleigh drag on the zonal flow approximately matches the vertically integrated total (mean plus eddy) angular momentum flux convergence at each latitude (Fig. 5).

The circulation statistics shown are computed from states sampled 4 times daily in the statistically steady states of the simulations. Statistics for the Jupiter simulation are computed from 1500 simulated days; statistics for the control simulations are computed from 900 simulated days. The statistics are first computed on the GCM's $\sigma$ surfaces, with the appropriate surface pressureweighting of the averages (e.g., Walker and Schneider 2006), and are then interpolated to pressure surfaces for display purposes. The divergence of eddy angular momentum fluxes is computed as the isobaric divergence of the eddy fluxes interpolated to pressure surfaces; this divergence does not differ significantly from the divergence of eddy angular momentum fluxes on $\sigma$ surfaces.

\section{h. Sensitivity to Rayleigh drag coefficient}

If the Rayleigh drag coefficient $k_{0}(\phi)$ is taken to be constant in latitude and equal to the value we use outside the equatorial region $\left(0.05 \mathrm{day}^{-1}\right)$, much higher intrinsic heat fluxes are needed to generate equatorial superrotation. In computationally less demanding simulations of a planet with four times Earth's radius but Jovian parameters otherwise (as in Table B1), we found that the intrinsic heat fluxes had to be of order $100 \mathrm{~W} \mathrm{~m}^{-2}$ to produce equatorial superrotation for a constant drag coefficient of $0.05 \mathrm{day}^{-1}$. If a smaller constant drag coefficient is chosen $\left(\leqslant 0.001\right.$ day $\left.^{-1}\right)$, 
equatorial superrotation can be generated with intrinsic heat fluxes comparable to $5.7 \mathrm{~W} \mathrm{~m}^{-2}$, but then the offequatorial jets are much wider and stronger than Jupiter's. The drag in the equatorial region has to be sufficiently weak to produce equatorial superrotation with the observed intrinsic heat flux, and the drag outside the equatorial region has to be sufficiently strong to reproduce the speeds and widths of Jupiter's off-equatorial jets.

\section{REFERENCES}

Andrews, D. G., and M. E. McIntyre, 1976: Planetary waves in horizontal and vertical shear: The generalized Eliassen-Palm relation and the mean zonal acceleration. J. Atmos. Sci., 33, 2031-2048

— Lagrangian-mean flow. J. Fluid Mech., 89, 609-646.

Atkinson, D. H., J. B. Pollack, and A. Seiff, 1998: The Galileo Probe Doppler Wind Experiment: Measurement of the deep zonal winds on Jupiter. J. Geophys. Res., 103, 22 911-22 928.

Betts, A. K., 1986: A new convective adjustment scheme. Part I: Observational and theoretical basis. Quart. J. Roy. Meteor. Soc., 112, 677-691.

, and M. J. Miller, 1986: A new convective adjustment scheme. Part II: Single column tests using GATE wave, BOMEX, ATEX and arctic air-mass data sets. Quart. J. Roy. Meteor. Soc., 112, 693-709.

Boer, G. J., 1983: Homogeneous and isotropic turbulence on the sphere. J. Atmos. Sci., 40, 154-163.

—, and T. G. Shepherd, 1983: Large-scale two-dimensional turbulence in the atmosphere. J. Atmos. Sci., 40, 164-184.

Bourke, W., 1974: A multilevel spectral model. I. Formulation and hemispheric integrations. Mon. Wea. Rev., 102, 687-701.

Busse, F. H., 1976: A simple model of convection in the Jovian atmosphere. Icarus, 29, 255-260.

1994: Convection-driven zonal flows and vortices in the major planets. Chaos, 4, 123-134.

Charney, J. G., 1963: A note on large-scale motions in the tropics. J. Atmos. Sci., 20, 607-609.

Conrath, B., P. Gierasch, and E. Ustinov, 1998: Thermal structure and para hydrogen fraction on the outer planets from Voyager IRIS measurements. Icarus, 135, 501-517.

Danilov, S., and D. Gurarie, 2002: Rhines scale and spectra of the $\beta$-plane turbulence with bottom drag. Phys. Rev. E, 65, 067301, doi:10.1103/PhysRevE.65.067301.

Donivan, F. F., and T. D. Carr, 1969: Jupiter's decametric rotation period. Astrophys. J., 157, L65-L68.

Dritschel, D. G., and M. E. McIntyre, 2008: Multiple jets as PV staircases: The Phillips effect and the resilience of eddytransport barriers. J. Atmos. Sci., 65, 855-874.

Durran, D. R., 1999: Numerical Methods for Wave Equations in Geophysical Fluid Dynamics. Vol. 32, Texts in Applied Mathematics, Springer, 483 pp.

Edmon, H. J., B. J. Hoskins, and M. E. McIntyre, 1980: EliassenPalm cross sections for the troposphere. J. Atmos. Sci., 37, 2600-2616.

Emanuel, K. A., J. D. Neelin, and C. S. Bretherton, 1994: On largescale circulations in convecting atmospheres. Quart. J. Roy. Meteor. Soc., 120, 1111-1143.

Gierasch, P. J., and Coauthors, 2000: Observation of moist convection in Jupiter's atmosphere. Nature, 403, 628-630.
Gill, A. E., 1982: Atmosphere-Ocean Dynamics. International Geophysics Series, Vol. 30, Academic Press, 662 pp.

Grote, E., and F. H. Busse, 2001: Dynamics of convection and dynamos in rotating spherical fluid shells. Fluid Dyn. Res., 28, 349-368.

Guillot, T., 1999: A comparison of the interiors of Jupiter and Saturn. Planet. Space Sci., 47, 1183-1200.

2005: The interiors of giant planets: Models and outstanding questions. Annu. Rev. Earth Planet. Sci., 33, 493-530.

—, D. J. Stevenson, W. B. Hubbard, and D. Saumon, 2004: The interior of Jupiter. Jupiter: The Planet, Satellites and Magnetosphere, F. Bagenal et al., Eds., Cambridge University Press, 35-57.

Hanel, R. A., B. J. Conrath, L. W. Herath, V. G. Kunde, and J. A. Pirraglia, 1981: Albedo, internal heat, and energy balance of Jupiter-Preliminary results of the Voyager infrared investigation. J. Geophys. Res., 86, 8705-8712.

Haynes, P. H., C. J. Marks, M. E. McIntyre, T. G. Shepherd, and K. P. Shine, 1991: On the "downward control" of extratropical diabatic circulations by eddy-induced mean zonal forces. J. Atmos. Sci., 48, 651-679.

Heimpel, M., and J. Aurnou, 2007: Turbulent convection in rapidly rotating spherical shells: A model for equatorial and highlatitude jets on Jupiter and Saturn. Icarus, 187, 540-557.

,$--\ldots$, and J. Wicht, 2005: Simulation of equatorial and highlatitude jets on Jupiter in a deep convection model. Nature, 438, 193-196.

Held, I. M., 1975: Momentum transport by quasi-geostrophic eddies. J. Atmos. Sci., 32, 1494-1497.

, 1999: Equatorial superrotation in Earth-like atmospheric models. Bernhard Haurwitz Memorial Lecture, 23 pp. [Available online at http://www.gfdl.gov/ ih/papers/super.ps.]

_ , and B. J. Hoskins, 1985: Large-scale eddies and the general circulation of the troposphere. Advances in Geophysics, Vol. 28A, Academic Press, 3-31.

_ of the dynamical cores of atmospheric general circulation models. Bull. Amer. Meteor. Soc., 75, 1825-1830.

Hide, R., 1969: Dynamics of the atmospheres of the major planets with an appendix on the viscous boundary layer at the rigid bounding surface of an electrically conducting rotating fluid in the presence of a magnetic field. J. Atmos. Sci., 26, 841853.

Ingersoll, A. P., 1990: Atmospheric dynamics of the outer planets. Science, 248, 308-315.

— , and D. Pollard, 1982: Motion in the interiors and atmospheres of Jupiter and Saturn: Scale analysis, anelastic equations, barotropic stability criterion. Icarus, 52, 62-80.

- and H. Kanamori, 1995: Waves from the collisions of comet Shoemaker-Levy 9 with Jupiter. Nature, 374, 706-708.

—, R. F. Beebe, J. L. Mitchell, G. W. Garneau, G. M. Yagi, and J.-P. Müller, 1981: Interaction of eddies and mean zonal flow on Jupiter as inferred from Voyager 1 and 2 images. J. Geophys. Res., 86, 8733-8743.

— , and Coauthors, 2004: Dynamics of Jupiter's atmosphere. Jupiter: The Planet, Satellites and Magnetosphere, F. Bagenal et al., Eds., Cambridge University Press, 105-128.

Kaspi, Y., 2008: Turbulent convection in an anelastic rotating sphere: A model for the circulation on the giant planets. Ph.D. thesis, Massachusetts Institute of Technology, $214 \mathrm{pp}$.

— , and G. R. Flierl, 2007: Formation of jets by baroclinic instability on gas planet atmospheres. J. Atmos. Sci., 64, 31773194. 
Kuo, H. L., 1951: Vorticity transfer as related to the development of the general circulation. J. Meteor., 8, 307-315.

Levine, J., D. Kraemer, and W. Kuhn, 1977: Solar radiation incident on Mars and the outer planets: Latitudinal, seasonal, and atmospheric effects. Icarus, 31, 136-145.

Liu, J. J., P. M. Goldreich, and D. J. Stevenson, 2008: Constraints on deep-seated zonal winds inside Jupiter and Saturn. Icarus, 196, 653-664.

Lodders, K., and B. Fegley Jr., 1998: The Planetary Scientist's Companion. Oxford University Press, 391 pp.

Lorenz, E. N., 1955: Available potential energy and the maintenance of the general circulation. Tellus, 7, 157-167.

Magalhães, J. A., A. Seiff, and R. E. Young, 2002: The stratification of Jupiter's troposphere at the Galileo probe entry site. Icarus, 158, 410-433.

Marston, J. B., E. Conover, and T. Schneider, 2008: Statistics of an unstable barotropic jet from a cumulant expansion. J. Atmos. Sci., 65, 1955-1966.

Matsuno, T., 1966: Quasi-geostrophic motions in the equatorial area. J. Meteor. Soc. Japan, 44, 25-42.

McIntyre, M., 1980: An introduction to the generalized Lagrangian-mean description of wave, mean-flow interaction. Pure Appl. Geophys., 118, 152-176.

_- 1982: How well do we understand the dynamics of stratospheric warmings? J. Meteor. Soc. Japan, 60, 37-65.

Nellis, W. J., S. T. Weir, and A. C. Mitchell, 1996: Metallization and electrical conductivity of hydrogen in Jupiter. Science, 273, 936-938.

O'Gorman, P. A., and T. Schneider, 2007: Recovery of atmospheric flow statistics in a general circulation model without nonlinear eddy-eddy interactions. Geophys. Res. Lett., 34, L22801, doi:10.1029/2007GL031779.

— , and —_, 2008: Weather-layer dynamics of baroclinic eddies and multiple jets in an idealized general circulation model. $J$. Atmos. Sci., 65, 524-535.

Peixoto, J. P., and A. H. Oort, 1992: Physics of Climate. American Institute of Physics, $555 \mathrm{pp}$.

Petty, G. W., 2006: A First Course in Atmospheric Radiation. 2nd ed. Sundog Publishing, 470 pp.

Plumb, R. A., 1979: Eddy fluxes of conserved quantities by smallamplitude waves. J. Atmos. Sci., 36, 1699-1704.

Porco, C., and Coauthors, 2003: Cassini imaging of Jupiter's atmosphere, satellites, and rings. Science, 299, 1541-1547.

Randel, W. J., and I. M. Held, 1991: Phase speed spectra of transient eddy fluxes and critical layer absorption. J. Atmos. Sci., 48, 688-697.

Read, P. L., P. J. Gierasch, B. J. Conrath, A. Simon-Miller, T. Fouchet, and Y. H. Yamazaki, 2006: Mapping potential-vorticity dynamics on Jupiter. I. Zonal-mean circulation from Cassini and Voyager 1 data. Quart. J. Roy. Meteor. Soc., 132, 1577-1603.

Rhines, P. B., 1975: Waves and turbulence on a beta-plane. J. Fluid Mech., 69, 417-443.

—, 1994: Jets. Chaos, 4, 313-339.

Salyk, C., A. P. Ingersoll, J. Lorre, A. Vasavada, and A. D. Del Genio, 2006: Interaction between eddies and mean flow in Jupiter's atmosphere: Analysis of Cassini imaging data. Icarus, 185, 430-442.

Sánchez-Lavega, A., and Coauthors, 2008: Depth of a strong Jovian jet from a planetary-scale disturbance driven by storms. Nature, 451, 437-440.

Saravanan, R., 1993: Equatorial superrotation and maintenance of the general circulation in two-level models. J. Atmos. Sci., 50, $1211-1227$.
Sardeshmukh, P. D., and B. J. Hoskins, 1988: The generation of global rotational flow by steady idealized tropical divergence. J. Atmos. Sci., 45, 1228-1251.

Schneider, E. K., 1977: Axially symmetric steady-state models of the basic state for instability and climate studies. Part II. Nonlinear calculations. J. Atmos. Sci., 34, 280-296.

Schneider, T., 2006: The general circulation of the atmosphere. Annu. Rev. Earth Planet. Sci., 34, 655-688.

and C. C. Walker, 2006: Self-organization of atmospheric macroturbulence into critical states of weak nonlinear eddyeddy interactions. J. Atmos. Sci., 63, 1569-1586.

$\longrightarrow$, and — 2008: Scaling laws and regime transitions of macroturbulence in dry atmospheres. J. Atmos. Sci., 65, $2153-$ 2173

Shepherd, T. G., 1987a: A spectral view of nonlinear fluxes and stationary-transient interaction in the atmosphere. J. Atmos. Sci., 44, 1166-1178.

_ 1987b: Rossby waves and two-dimensional turbulence in a large-scale zonal jet. J. Fluid Mech., 183, 467-509.

Showman, A., and A. Ingersoll, 1998: Interpretation of Galileo probe data and implications for Jupiter's dry downdrafts. Icarus, 132, 205-220.

Simon-Miller, A. A., B. J. Conrath, P. J. Gierasch, G. S. Orton, R. K. Achterberg, F. M. Flasar, and B. M. Fisher, 2006: Jupiter's atmospheric temperatures: From Voyager IRIS to Cassini CIRS. Icarus, 180, 98-112.

Smith, K. S., G. Boccaletti, C. C. Henning, I. N. Marinov, C. Y. Tam, I. M. Held, and G. K. Vallis, 2002: Turbulent diffusion in the geostrophic inverse cascade. J. Fluid Mech., 469, 13-48.

Sobel, A. H., J. Nilsson, and L. M. Polvani, 2001: The weak temperature gradient approximation and balanced tropical moisture waves. J. Atmos. Sci., 58, 3650-3665.

Sromovsky, L. A., and P. M. Fry, 2002: Jupiter's cloud structure as constrained by Galileo probe and HST observations. Icarus, 157, 373-400.

, A. D. Collard, P. M. Fry, G. S. Orton, M. T. Lemmon, M. G. Tomasko, and R. S. Freedman, 1998: Galileo probe measurements of thermal and solar radiation fluxes in the Jovian atmosphere. J. Geophys. Res., 103, 22 929-22 977.

Suarez, M. J., and D. G. Duffy, 1992: Terrestrial superrotation: A bifurcation of the general circulation. J. Atmos. Sci., 49, 1541-1554.

Sun, Z.-P., G. Schubert, and G. A. Glatzmaier, 1993: Banded surface flow maintained by convection in a model of the rapidly rotating giant planets. Science, 260, 661-664.

Vallis, G. K., 2006: Atmospheric and Oceanic Fluid Dynamics: Fundamentals and Large-Scale Circulation. Cambridge University Press, $771 \mathrm{pp}$.

Vasavada, A. R., and A. P. Showman, 2005: Jovian atmospheric dynamics: An update after Galileo and Cassini. Rep. Prog. Phys., 68, 1935-1996.

Walker, C. C., and T. Schneider, 2006: Eddy influences on Hadley circulations: Simulations with an idealized GCM. J. Atmos. Sci., 63, 3333-3350.

West, R. A., K. H. Baines, A. J. Friedson, D. Banfield, B. Ragent, and F. W. Taylor, 2004: Jovian clouds and haze. Jupiter: The Planet, Satellites and Magnetosphere, F. Bagenal et al., Eds., Cambridge University Press, 79-104.

Williams, G. P., 1979: Planetary circulations: 2. The Jovian quasigeostrophic regime. J. Atmos. Sci., 36, 932-968.

_ 2002: Jovian dynamics. Part II: The genesis and equilibration of vortex sets. J. Atmos. Sci., 59, 1356-1370.

2003: Jovian dynamics. Part III: Multiple, migrating, and equatorial jets. J. Atmos. Sci., 60, 1270-1296. 